\title{
The generalized viscosity implicit rule of nonexpansive semigroup in Banach spaces
}

\author{
Chaichana Jaiboon ${ }^{\mathrm{a}}$, Somyot Plubtieng ${ }^{\mathrm{b}}$, Phayap Katchang ${ }^{\mathrm{c}, *}$ \\ ${ }^{a}$ Department of Mathematics, Faculty of Liberal Arts, Rajamangala University of Technology Rattanakosin, Nakhon Pathom 73170, \\ Thailand. \\ ${ }^{b}$ Department of Mathematics, Faculty of Science, Naresuan University, Phitsanulok 65000, Thailand. \\ ${ }^{c}$ Division of Mathematics, Faculty of Science and Agricultural Technology, Rajamangala University of Technology Lanna Tak, Tak 63000, \\ Thailand.
}

Communicated by Y. J. Cho

\begin{abstract}
In this research, we focus on a common fixed point problem of a nonexpansive semigroup with the generalized viscosity methods for implicit iterative algorithms. Our main objective is to construct the new strong convergence theorems under certain appropriate conditions in uniformly convex and uniformly smooth Banach spaces. Specifically, the main results make a contribution to the implicit midpoint theorems. The findings for theorems in Hilbert spaces and the other forms of a nonexpansive semigroup can be used in several practical purposes. Finally, a numerical example in 3 dimensions is provided to support our main results.
\end{abstract}

Keywords: Nonexpansive semigroup, fixed point, generalized viscosity, implicit, Banach space.

2010 MSC: 47H10, 47H20, 47J20.

(C)2018 All rights reserved.

\section{Introduction}

Let $E$ be a real Banach space, $C$ be a nonempty closed convex subset of $E$, and $E^{*}$ be the dual space of $E$ with norm $\|\cdot\|$ and $\langle\cdot, \cdot\rangle$ pairing between $E$ and $E^{*}$.

- The duality mapping $\mathrm{J}: \mathrm{E} \rightarrow 2^{\mathrm{E}^{*}}$ is defined by

$$
J(x)=\left\{x^{*} \in E^{*}:\left\langle x, x^{*}\right\rangle=\|x\|^{2},\left\|x^{*}\right\|=\|x\|\right\}
$$

for all $x \in E$. It is well known that if $E$ is a Hilbert space then $J$ is the identity mapping, and if $E$ is smooth then $\mathrm{J}$ is single-valued.

\footnotetext{
*Corresponding author

Email addresses: chaichana.jai@rmutr.ac.th (Chaichana Jaiboon), somyotp@nu.ac.th (Somyot Plubtieng),

p.katchang@rmutl.ac.th (Phayap Katchang)
}

doi: $10.22436 /$ jnsa.011.06.02

Received: 2017-06-29 Revised: 2018-03-22 Accepted: 2018-03-22 
- A mapping $\mathrm{T}: \mathrm{C} \rightarrow \mathrm{C}$ is called a nonexpansive, if

$$
\|\mathrm{T} x-\mathrm{T} y\| \leqslant\|x-y\|, \forall x, y \in C
$$

and $F(T)=\{x \in C: T x=x\}$ is the set of fixed points of $T$.

- A mapping $\mathrm{f}: \mathrm{C} \rightarrow \mathrm{C}$ is called a contraction, if there exists $\alpha \in(0,1)$ such that

$$
\|f(x)-f(y)\| \leqslant \alpha\|x-y\|, \forall x, y \in C .
$$

- A family $\mathcal{S}=\{\mathrm{S}(\mathrm{s}): 0 \leqslant s<\infty\}$ of mappings of $\mathrm{C}$ into itself is called a nonexpansive semigroup on $\mathrm{C}$ if it satisfies the following conditions:

(i) $S(0) x=x$ for all $x \in C$;

(ii) $S(s+t)=S(s) S(t)$ for all $s, t \geqslant 0$;

(iii) $\|S(s) x-S(s) y\| \leqslant\|x-y\|$ for all $x, y \in C$ and $s \geqslant 0$;

(iv) for each $x \in C$, the mapping $S(\cdot) x$ from $[0, \infty)$ into $C$ is continuous.

$\mathcal{S}$, i.e., $\mathrm{F}(\mathcal{S})=\{x \in C: S(s) x=x, \forall s>0\}$ is a common fixed point set of a nonexpansive semigroup. It is easy to see that $F(\mathcal{S})$ is closed and convex (see also $[3,5,6,11]$ ).

Definition 1.1. A mapping $\psi: \mathbb{R}^{+} \rightarrow \mathbb{R}^{+}$is called an $L$-function if $\psi(0)=0, \psi(t)>0, \forall t>0$ and for every $s>0$ there exists $u>s$ such that

$$
\psi(t) \leqslant s \text { for } t \in[s, u] .
$$

Note that every $L$-function $\psi$ satisfies $\psi(t)<t, \forall t>0$.

Definition 1.2. Let $(X, d)$ be a metric space. A mapping $f: X \rightarrow X$ is said to be:

(i) $(\psi, L)$-contraction if $\psi: \mathbb{R}^{+} \rightarrow \mathbb{R}^{+}$is an $L$-function and $d(f(x), f(y))<\psi(d(x, y))$ for all $x, y \in X$ with $x \neq y$;

(ii) Meir-Keeler type mapping if for each $\epsilon>0$ there exists $\delta=\delta(\epsilon)>0$ such that for each $x, y \in X$ with $d(x, y)<\epsilon+\delta$ we have $d(f(x), f(y))<\epsilon$.

Theorem 1.3 ([7]). Let $(\mathrm{X}, \mathrm{d})$ be a metric space and $\mathrm{f}: \mathrm{X} \rightarrow \mathrm{X}$ a mapping. Then the following assertions are equivalent:

(i) $\mathrm{f}$ is a Meir-Keeler type mapping;

(ii) there exists an L-function $\psi: \mathbb{R}^{+} \rightarrow \mathbb{R}^{+}$such that $\mathrm{f}$ is a $(\psi, \mathrm{L})$-contraction.

Proposition 1.4 ([10]). Let $\mathrm{C}$ be a convex subset of a Banach space $\mathrm{E}$. Let $\mathrm{f}: \mathrm{C} \rightarrow \mathrm{C}$ be a Meir-Keeler type mapping. Then for each $\epsilon>0$ there exists $r \in(0,1)$ such that for each $x, y \in C$ with $\|x-y\| \geqslant \epsilon$, we have

$$
\|f(x)-f(y)\| \leqslant r\|x-y\| .
$$

Now, a Meir-Keeler type mapping or $(\psi, \mathrm{L})$-contraction is called generalized contraction mapping. We suppose that the function $\psi$ based on the definition of the $(\psi, L)$-contraction is continuous and strictly increasing and $\lim _{t \rightarrow \infty} \eta(t)=\infty$, where $\eta(t)=t-\psi(t), t \in \mathbb{R}^{+}$. In consequence, we have that $\eta$ is a bijection on $\mathbb{R}^{+}$.

In 2015, the viscosity implicit midpoint theorem in Hilbert spaces was introduced by Xu et al. [13]:

$$
x_{n+1}=\alpha_{n} f\left(x_{n}\right)+\left(1-\alpha_{n}\right) T\left(\frac{x_{n}+x_{n+1}}{2}\right), n \geqslant 0 .
$$

In the same year, Ke et al. [4] introduced the two viscosity implicit midpoint theorem in Hilbert spaces:

$$
x_{n+1}=\alpha_{n} Q\left(x_{n}\right)+\left(1-\alpha_{n}\right) T\left(s_{n} x_{n}+\left(1-s_{n}\right) x_{n+1}\right),
$$


and

$$
x_{n+1}=\alpha_{n} x_{n}+\beta_{n} Q\left(x_{n}\right)+\gamma_{n} T\left(s_{n} x_{n}+\left(1-s_{n}\right) x_{n+1}\right) .
$$

One year later, Yan et al. [14] introduced an implicit iteration for a generalized contraction mapping in Banach space:

$$
x_{n+1}=\alpha_{n} x_{n}+\beta_{n} f\left(x_{n}\right)+\gamma_{n} T\left(s_{n} x_{n}+\left(1-s_{n}\right) x_{n+1}\right) .
$$

They proved the strong convergence theorems.

Motivated and inspired by the idea of Yan et al. [14], we introduce the new implicit iterative scheme and the new implicit midpoint rule with viscosity approximation method based on a generalized contraction mapping for finding solutions of fixed point problems for nonexpansive semigroup. We shall prove the strong convergence theorems in uniformly convex and uniformly smooth Banach space under some parameters controlling conditions. Our results extend and improve the recent results of Yan et al. [14] and other authors.

\section{Preliminaries}

According to our framework throughout this research, we first preview some definitions involving a Banach space $\mathrm{E}$ as follows. Let $\mathrm{U}=\{\mathrm{x} \in \mathrm{E}:\|x\|=1\}$.

- $E$ is said to be uniformly convex if, for any $\epsilon \in(0,2]$, there exists $\delta>0$ such that, for any $x, y \in U$, $\|x-y\| \geqslant \epsilon$ implies $\left\|\frac{x+y}{2}\right\| \leqslant 1-\delta$.

It is known that a uniformly convex Banach space is reflexive and strictly convex.

- $E$ is said to be smooth if $\lim _{t \rightarrow 0} \frac{\|x+t y\|-\|x\|}{t}$ exists for all $x, y \in U$.

It is also said to be uniformly smooth if the limit is attained uniformly for all $x, y \in \mathrm{U}$. The modulus of smoothness of $E$ is defined by

$$
\rho(\tau)=\sup \left\{\frac{1}{2}(\|x+y\|+\|x-y\|)-1: x, y \in E,\|x\|=1,\|y\|=\tau\right\},
$$

where $\rho:[0, \infty) \rightarrow[0, \infty)$ is a function.

It is known that $E$ is uniformly smooth if and only if $\lim _{\tau \rightarrow 0} \frac{\rho(\tau)}{\tau}=0$.

A Banach space $E$ is said to satisfy Opial's condition if for any sequence $\left\{x_{n}\right\}$ in $E, x_{n} \rightarrow x(n \rightarrow \infty)$ implies

$$
\limsup _{n \rightarrow \infty}\left\|x_{n}-x\right\|<\limsup _{n \rightarrow \infty}\left\|x_{n}-y\right\|, \forall y \in E \text { with } x \neq y .
$$

By [2, Theorem 1], it is well known that if E admits a weakly sequentially continuous duality mapping, then E satisfies Opial's condition, and E is smooth.

The following lemmas are very useful for proving our main results.

Lemma 2.1 ([9]). Let $\left\{x_{n}\right\}$ and $\left\{y_{n}\right\}$ be bounded sequences in a Banach space $X$ and let $\left\{\beta_{n}\right\}$ be a sequence in $[0,1]$ with $0<\liminf _{n \rightarrow \infty} \beta_{n} \leqslant \limsup _{n \rightarrow \infty} \beta_{n}<1$. Suppose $x_{n+1}=\left(1-\beta_{n}\right) y_{n}+\beta_{n} x_{n}$ for all integers $n \geqslant 0$ and $\lim \sup _{n \rightarrow \infty}\left(\left\|y_{n+1}-y_{n}\right\|-\left\|x_{n+1}-x_{n}\right\|\right) \leqslant 0$. Then, $\lim _{n \rightarrow \infty}\left\|y_{n}-x_{n}\right\|=0$.

Lemma 2.2 ([8]). Let $\mathrm{C}$ be a nonempty closed and convex subset of a uniformly smooth Banach space E. Let $\mathrm{T}: \mathrm{C} \rightarrow \mathrm{C}$ be a nonexpansive mapping such that $\mathrm{F}(\mathrm{T}) \neq \emptyset$ and $\mathrm{f}: \mathrm{C} \rightarrow \mathrm{C}$ be a generalized contraction mapping. Then $\left\{x_{\mathrm{t}}\right\}$ defined by $\mathrm{x}_{\mathrm{t}}=\mathrm{tf}\left(\mathrm{x}_{\mathrm{t}}\right)+(1-\mathrm{t}) \mathrm{T} \mathrm{x}_{\mathrm{t}}$ for $\mathrm{t} \in(0,1)$, converges strongly to $\mathrm{x}^{*} \in \mathrm{F}(\mathrm{T})$ as $\mathrm{t} \rightarrow 0$, which solves the variational inequality:

$$
\left\langle\mathrm{f}\left(x^{*}\right)-x^{*}, \mathrm{~J}\left(z-x^{*}\right)\right\rangle \leqslant 0, \forall z \in \mathrm{F}(\mathrm{T}) .
$$


Lemma 2.3 ([8]). Let $\mathrm{C}$ be a nonempty closed and convex subset of a uniformly smooth Banach space $\mathrm{E}$. Let $\mathrm{T}: \mathrm{C} \rightarrow \mathrm{C}$ be a nonexpansive mapping such that $\mathrm{F}(\mathrm{T}) \neq \emptyset$ and $\mathrm{f}: \mathrm{C} \rightarrow \mathrm{C}$ be a generalized contraction mapping. Assume that $\left\{\mathrm{x}_{\mathrm{t}}\right\}$ defined by $\mathrm{x}_{\mathrm{t}}=\mathrm{tf}\left(\mathrm{x}_{\mathrm{t}}\right)+(1-\mathrm{t}) \mathrm{T} \mathrm{x}_{\mathrm{t}}$, converges strongly to $\mathrm{x}^{*} \in \mathrm{F}(\mathrm{T})$ as $\mathrm{t} \rightarrow 0$. Suppose that $\left\{x_{n}\right\}$ is bounded sequence such that $x_{n}-T x_{n} \rightarrow 0$ as $n \rightarrow \infty$. Then

$$
\limsup _{n \rightarrow \infty}\left\langle f\left(x^{*}\right)-x^{*}, J\left(x_{n}-x^{*}\right)\right\rangle \leqslant 0 .
$$

Lemma 2.4 ([12]). Assume $\left\{a_{n}\right\}$ is a sequence of nonnegative real numbers such that

$$
a_{n+1} \leqslant\left(1-\alpha_{n}\right) a_{n}+\delta_{n}, n \geqslant 0,
$$

where $\left\{\alpha_{n}\right\}$ is a sequence in $(0,1)$ and $\left\{\delta_{n}\right\}$ is a sequence in $\mathbb{R}$ such that

(1) $\sum_{n=1}^{\infty} \alpha_{n}=\infty$;

(2) $\limsup _{n \rightarrow \infty} \frac{\delta_{n}}{\alpha_{n}} \leqslant 0$ or $\sum_{n=1}^{\infty}\left|\delta_{n}\right|<\infty$.

Then $\lim _{n \rightarrow \infty} a_{n}=0$.

\section{Main results}

Theorem 3.1. Let $\mathrm{C}$ be a nonempty closed convex subset of uniformly convex and uniformly smooth Banach space $\mathrm{E}$. Let $\mathrm{f}$ be a generalized contraction mapping from $\mathrm{C}$ into itself and $\mathcal{S}=\{\mathrm{T}(\mathrm{t}): \mathrm{t} \geqslant 0\}$ be a nonexpansive semigroup from $\mathrm{C}$ into itself such that $\mathrm{F}(\mathcal{S}) \neq 0$. Let $\left\{\mathrm{x}_{\mathrm{n}}\right\}$ be the sequences defined by $\mathrm{x}_{1} \in \mathrm{C}$ and

$$
\left\{\begin{array}{l}
z_{n}=\lambda_{n} x_{n}+\left(1-\lambda_{n}\right) x_{n+1} \\
y_{n}=\delta_{n} f\left(x_{n}\right)+\left(1-\delta_{n}\right) z_{n} \\
x_{n+1}=\alpha_{n} x_{n}+\beta_{n} f\left(x_{n}\right)+\gamma_{n} T\left(\mu_{n}\right) y_{n},
\end{array}\right.
$$

where $\left\{\alpha_{n}\right\},\left\{\beta_{n}\right\},\left\{\gamma_{n}\right\},\left\{\delta_{n}\right\}$, and $\left\{\lambda_{n}\right\}$ are the sequences in $(0,1)$. The following conditions are satisfied:

(i) $\alpha_{\mathrm{n}}+\beta_{\mathrm{n}}+\gamma_{\mathrm{n}}=1$;

(ii) $\lim _{n \rightarrow \infty} \beta_{n}=\lim _{n \rightarrow \infty} \delta_{n}=\lim _{n \rightarrow \infty} \mu_{n}=\lim _{n \rightarrow \infty}\left|\alpha_{n+1}-\alpha_{n}\right|=\lim _{n \rightarrow \infty}\left|\lambda_{n+1}-\lambda_{n}\right|=0$ and $\lim _{n \rightarrow \infty} \sup _{x \in \tilde{C}} \| T\left(\mu_{n+1}\right) x-$ $\mathrm{T}\left(\mu_{\mathrm{n}}\right) \mathrm{x} \|=0$, where $\tilde{\mathrm{C}}$ is a bounded subset of $\mathrm{C}$;

(iii) $0<\liminf _{n \rightarrow \infty} \alpha_{n} \leqslant \limsup _{n \rightarrow \infty} \alpha_{n}<1$;

(iv) $\sum_{n=0}^{\infty} \beta_{n}=\infty$ for all $n \geqslant 1$.

Then $\left\{x_{n}\right\}$ converges strongly to $x^{*} \in F(S)$ which also solves the following variational inequality:

$$
\left\langle(\mathrm{I}-\mathrm{f}) \chi^{*}, \mathrm{~J}\left(z-x^{*}\right)\right\rangle \leqslant 0, \forall z \in \mathrm{F}(\mathcal{S}) .
$$

Proof. First of all, we prove that $\left\{x_{n}\right\}$ is bounded. Let $p \in F(\mathcal{S})$, we have

$$
\begin{aligned}
\left\|y_{n}-p\right\| & \leqslant \delta_{n}\left\|f\left(x_{n}\right)-p\right\|+\left(1-\delta_{n}\right)\left\|z_{n}-p\right\| \\
& \leqslant \delta_{n}\left\|f\left(x_{n}\right)-f(p)\right\|+\delta_{n}\|f(p)-p\|+\left(1-\delta_{n}\right)\left\|z_{n}-p\right\| \\
& \leqslant \delta_{n} \psi\left\|x_{n}-p\right\|+\delta_{n}\|f(p)-p\|+\left(1-\delta_{n}\right)\left[\lambda_{n}\left\|x_{n}-p\right\|+\left(1-\lambda_{n}\right)\left\|x_{n+1}-p\right\|\right] \\
& =\left[\delta_{n} \psi+\left(1-\delta_{n}\right) \lambda_{n}\right]\left\|x_{n}-p\right\|+\delta_{n}\|f(p)-p\|+\left(1-\delta_{n}\right)\left(1-\lambda_{n}\right)\left\|x_{n+1}-p\right\|
\end{aligned}
$$

and

$$
\begin{aligned}
\left\|x_{n+1}-p\right\| & \leqslant \alpha_{n}\left\|x_{n}-p\right\|+\beta_{n}\left\|f\left(x_{n}\right)-p\right\|+\gamma_{n}\left\|T\left(\mu_{n}\right) y_{n}-p\right\| \\
& \leqslant \alpha_{n}\left\|x_{n}-p\right\|+\beta_{n}\left\|f\left(x_{n}\right)-f(p)\right\|+\beta_{n}\|f(p)-p\|+\gamma_{n}\left\|y_{n}-p\right\|
\end{aligned}
$$




$$
\begin{aligned}
\leqslant & \alpha_{n}\left\|x_{n}-p\right\|+\beta_{n} \psi\left\|x_{n}-p\right\|+\beta_{n}\|f(p)-p\| \\
& +\gamma_{n}\left\{\left[\delta_{n} \psi+\left(1-\delta_{n}\right) \lambda_{n}\right]\left\|x_{n}-p\right\|+\delta_{n}\|f(p)-p\|+\left(1-\delta_{n}\right)\left(1-\lambda_{n}\right)\left\|x_{n+1}-p\right\|\right\} \\
= & {\left[\alpha_{n}+\beta_{n} \psi+\gamma_{n} \delta_{n} \psi+\gamma_{n}\left(1-\delta_{n}\right) \lambda_{n}\right]\left\|x_{n}-p\right\|+\left(\beta_{n}+\gamma_{n} \delta_{n}\right)\|f(p)-p\| } \\
& +\gamma_{n}\left(1-\delta_{n}\right)\left(1-\lambda_{n}\right)\left\|x_{n+1}-p\right\| \\
= & {\left[1-\gamma_{n}\left(1-\delta_{n}\right)\left(1-\lambda_{n}\right)-\left(\beta_{n}+\gamma_{n} \delta_{n}\right)(1-\psi)\right]\left\|x_{n}-p\right\|+\left(\beta_{n}+\gamma_{n} \delta_{n}\right)\|f(p)-p\| } \\
& +\gamma_{n}\left(1-\delta_{n}\right)\left(1-\lambda_{n}\right)\left\|x_{n+1}-p\right\| \\
= & {\left[1-\gamma_{n}\left(1-\delta_{n}\right)\left(1-\lambda_{n}\right)-\left(\beta_{n}+\gamma_{n} \delta_{n}\right) \eta\right]\left\|x_{n}-p\right\|+\left(\beta_{n}+\gamma_{n} \delta_{n}\right) \eta \eta^{-1}\|f(p)-p\| } \\
& +\gamma_{n}\left(1-\delta_{n}\right)\left(1-\lambda_{n}\right)\left\|x_{n+1}-p\right\|, \\
\left\|x_{n+1}-p\right\| \leqslant & \alpha_{n}\left\|x_{n}-p\right\|+\beta_{n}\left\|f\left(x_{n}\right)-p\right\|+\gamma_{n}\left\|T\left(\mu_{n}\right) y_{n}-p\right\| \\
\leqslant & \alpha_{n}\left\|x_{n}-p\right\|+\beta_{n}\left\|f\left(x_{n}\right)-f(p)\right\|+\beta_{n}\|f(p)-p\|+\gamma_{n}\left\|y_{n}-p\right\| \\
\leqslant & \alpha_{n}\left\|x_{n}-p\right\|+\beta_{n} \psi\left\|x_{n}-p\right\|+\beta_{n}\|f(p)-p\| \\
& +\gamma_{n}\left\{\left[\delta_{n} \psi+\left(1-\delta_{n}\right) \lambda_{n}\right]\left\|x_{n}-p\right\|+\delta_{n}\|f(p)-p\|+\left(1-\delta_{n}\right)\left(1-\lambda_{n}\right)\left\|x_{n}+1-p\right\|\right\} \\
= & {\left[\alpha_{n}+\beta_{n} \psi+\gamma_{n} \delta_{n} \psi+\gamma_{n}\left(1-\delta_{n}\right) \lambda_{n}\right]\left\|x_{n}-p\right\|+\left(\beta_{n}+\gamma_{n} \delta_{n}\right)\|f(p)-p\| } \\
& +\gamma_{n}\left(1-\delta_{n}\right)\left(1-\lambda_{n}\right)\left\|x_{n+1}-p\right\| \\
= & {\left[1-\gamma_{n}\left(1-\delta_{n}\right)\left(1-\lambda_{n}\right)-\left(\beta_{n}+\gamma_{n} \delta_{n}\right)(1-\psi)\right]\left\|x_{n}-p\right\|+\left(\beta_{n}+\gamma_{n} \delta_{n}\right)\|f(p)-p\| } \\
& +\gamma_{n}\left(1-\delta_{n}\right)\left(1-\lambda_{n}\right)\left\|x_{n+1}-p\right\| \\
= & {\left[1-\gamma_{n}\left(1-\delta_{n}\right)\left(1-\lambda_{n}\right)-\left(\beta_{n}+\gamma_{n} \delta_{n}\right) \eta\right]\left\|x_{n}-p\right\|+\left(\beta_{n}+\gamma_{n} \delta_{n}\right) \eta \eta^{-1}\|f(p)-p\| } \\
& +\gamma_{n}\left(1-\delta_{n}\right)\left(1-\lambda_{n}\right)\left\|x_{n+1}-p\right\| .
\end{aligned}
$$

It follows that

$$
\left\|x_{n+1}-p\right\| \leqslant\left[1-\frac{\left(\beta_{n}+\gamma_{n} \delta_{n}\right) \eta}{1-\gamma_{n}\left(1-\delta_{n}\right)\left(1-\lambda_{n}\right)}\right]\left\|x_{n}-p\right\|+\frac{\left(\beta_{n}+\gamma_{n} \delta_{n}\right) \eta}{1-\gamma_{n}\left(1-\delta_{n}\right)\left(1-\lambda_{n}\right)} \eta^{-1}\|f(p)-p\| .
$$

By induction, we conclude that

$$
\left\|x_{n}-p\right\| \leqslant \max \left\{\left\|x_{1}-p\right\|, \eta^{-1}\|f(p)-p\|\right\}, \forall n \geqslant 0 .
$$

This implies that $\left\{x_{n}\right\}$ is bounded, so are $\left\{f\left(x_{n}\right)\right\},\left\{y_{n}\right\},\left\{T\left(\mu_{n}\right) y_{n}\right\}$, and $\left\{z_{n}\right\}$.

Next, we will show that $\lim _{n \rightarrow \infty}\left\|x_{n+1}-x_{n}\right\|=0$ and we observe that

$$
\begin{aligned}
\left\|z_{n+1}-z_{n}\right\|= & \left\|\left(\lambda_{n+1} x_{n+1}+\left(1-\lambda_{n+1}\right) x_{n+2}\right)-\left(\lambda_{n} x_{n}+\left(1-\lambda_{n}\right) x_{n+1}\right)\right\| \\
= & \| \lambda_{n+1} x_{n+1}-\lambda_{n+1} x_{n}+\lambda_{n+1} x_{n}+\left(1-\lambda_{n+1}\right) x_{n+2} \\
& -\left(1-\lambda_{n+1}\right) x_{n+1}+\left(1-\lambda_{n+1}\right) x_{n+1}-\lambda_{n} x_{n}-\left(1-\lambda_{n}\right) x_{n+1} \| \\
= & \left\|\lambda_{n+1}\left(x_{n+1}-x_{n}\right)+\left(\lambda_{n+1}-\lambda_{n}\right) x_{n}+\left(1-\lambda_{n+1}\right)\left(x_{n+2}-x_{n+1}\right)+\left(\lambda_{n}-\lambda_{n+1}\right) x_{n+1}\right\| \\
\leqslant & \lambda_{n+1}\left\|x_{n+1}-x_{n}\right\|+\left|\lambda_{n+1}-\lambda_{n}\right|\left(\left\|x_{n}\right\|+\left\|x_{n+1}\right\|\right)+\left(1-\lambda_{n+1}\right)\left\|x_{n+2}-x_{n+1}\right\|, \\
\left\|y_{n+1}-y_{n}\right\|= & \left\|\left(\delta_{n+1} f\left(x_{n+1}\right)+\left(1-\delta_{n+1}\right) z_{n+1}\right)-\left(\delta_{n} f\left(x_{n}\right)+\left(1-\delta_{n}\right) z_{n}\right)\right\| \\
= & \| \delta_{n+1} f\left(x_{n+1}\right)-\delta_{n+1} f\left(x_{n}\right)+\delta_{n+1} f\left(x_{n}\right)+\left(1-\delta_{n+1}\right) z_{n+1} \\
& -\left(1-\delta_{n+1}\right) z_{n}+\left(1-\delta_{n+1}\right) z_{n}-\delta_{n} f\left(x_{n}\right)-\left(1-\delta_{n}\right) z_{n} \| \\
= & \left\|\delta_{n+1}\left(f\left(x_{n+1}\right)-f\left(x_{n}\right)\right)+\left(\delta_{n+1}-\delta_{n}\right) f\left(x_{n}\right)+\left(1-\delta_{n+1}\right)\left(z_{n+1}-z_{n}\right)+\left(\delta_{n}-\delta_{n+1}\right) z_{n}\right\| \\
\leqslant & \delta_{n+1}\left\|f\left(x_{n+1}\right)-f\left(x_{n}\right)\right\|+\left|\delta_{n+1}-\delta_{n}\right|\left(\left\|f\left(x_{n}\right)\right\|+\left\|z_{n}\right\|\right)+\left(1-\delta_{n+1}\right)\left\|z_{n+1}-z_{n}\right\| \\
\leqslant & \delta_{n+1} \psi\left\|x_{n+1}-x_{n}\right\|+\left|\delta_{n+1}-\delta_{n}\right|\left(\left\|f\left(x_{n}\right)\right\|+\left\|z_{n}\right\|\right)+\left(1-\delta_{n+1}\right)\left[\lambda_{n+1}\left\|x_{n+1}-x_{n}\right\|\right. \\
& \left.+\left|\lambda_{n+1}-\lambda_{n}\right|\left(\left\|x_{n}\right\|+\left\|x_{n+1}\right\|\right)+\left(1-\lambda_{n+1}\right)\left\|x_{n+2}-x_{n+1}\right\|\right] \\
= & \left(\delta_{n+1} \psi+\left(1-\delta_{n+1}\right) \lambda_{n+1}\right)\left\|x_{n+1}-x_{n}\right\|+\left|\delta_{n+1}-\delta_{n}\right|\left(\left\|f\left(x_{n}\right)\right\|+\left\|z_{n}\right\|\right) \\
& +\left(1-\delta_{n+1}\right)\left|\lambda_{n+1}-\lambda_{n}\right|\left(\left\|x_{n}\right\|+\left\|x_{n+1}\right\|\right)+\left(1-\delta_{n+1}\right)\left(1-\lambda_{n+1}\right)\left\|x_{n+2}-x_{n+1}\right\|,
\end{aligned}
$$


and

$$
\begin{aligned}
& \left\|x_{n+2}-x_{n+1}\right\|=\|\left(\alpha_{n+1} x_{n+1}+\beta_{n+1} f\left(x_{n+1}\right)+\gamma_{n+1} T\left(\mu_{n+1}\right) y_{n+1}\right) \\
& -\left(\alpha_{n} x_{n}+\beta_{n} f\left(x_{n}\right)+\gamma_{n} T\left(\mu_{n}\right) y_{n}\right) \| \\
& =\| \alpha_{n+1} x_{n+1}-\alpha_{n+1} x_{n}+\alpha_{n+1} x_{n}+\beta_{n+1} f\left(x_{n+1}\right)-\beta_{n+1} f\left(x_{n}\right)+\beta_{n+1} f\left(x_{n}\right) \\
& +\gamma_{n+1} T\left(\mu_{n+1}\right) y_{n+1}-\gamma_{n+1} T\left(\mu_{n}\right) y_{n}+\gamma_{n+1} T\left(\mu_{n}\right) y_{n}-\alpha_{n} x_{n}-\beta_{n} f\left(x_{n}\right) \\
& -\gamma_{n} T\left(\mu_{n}\right) y_{n} \| \\
& =\| \alpha_{n+1}\left(x_{n+1}-x_{n}\right)+\beta_{n+1}\left(f\left(x_{n+1}\right)-f\left(x_{n}\right)\right)+\gamma_{n+1}\left(T\left(\mu_{n+1}\right) y_{n+1}-T\left(\mu_{n}\right) y_{n}\right) \\
& +\left(\alpha_{n+1}-\alpha_{n}\right) x_{n}+\left(\beta_{n+1}-\beta_{n}\right) f\left(x_{n}\right)+\left(\gamma_{n+1}-\gamma_{n}\right) T\left(\mu_{n}\right) y_{n} \| \\
& \leqslant \alpha_{n+1}\left\|x_{n+1}-x_{n}\right\|+\beta_{n+1}\left\|f\left(x_{n+1}\right)-f\left(x_{n}\right)\right\|+\gamma_{n+1}\left\|T\left(\mu_{n+1}\right) y_{n+1}-T\left(\mu_{n}\right) y_{n}\right\| \\
& +\left|\alpha_{n+1}-\alpha_{n}\right|\left\|x_{n}||+\left|\beta_{n+1}-\beta_{n}\right|\left|f\left(x_{n}\right)\right||+| \gamma_{n+1}-\gamma_{n} \mid\right\| T\left(\mu_{n}\right) y_{n} \| \\
& \leqslant \alpha_{n+1}\left\|x_{n+1}-x_{n}\right\|+\beta_{n+1} \psi\left\|x_{n+1}-x_{n}\right\| \\
& +\gamma_{n+1}\left(\left\|T\left(\mu_{n+1}\right) y_{n+1}-T\left(\mu_{n+1}\right) y_{n}\right\|+\left\|T\left(\mu_{n+1}\right) y_{n}-T\left(\mu_{n}\right) y_{n}\right\|\right) \\
& +\left|\alpha_{n+1}-\alpha_{n}\right||| x_{n}\left\|+\left|\beta_{n+1}-\beta_{n}\right||| f\left(x_{n}\right)\right\|+\left|\gamma_{n+1}-\gamma_{n}\right| \| T\left(\mu_{n}\right) y_{n}|| \\
& =\left(\alpha_{n+1}+\beta_{n+1} \psi\right)\left\|x_{n+1}-x_{n}\right\|+\gamma_{n+1} \text { sup }\left\|T\left(\mu_{n+1}\right) y-T\left(\mu_{n}\right) y\right\| \\
& y \in\left\{y_{n}\right\} \\
& +\left|\alpha_{n+1}-\alpha_{n}\right||| x_{n}||+\left|\beta_{n+1}-\beta_{n}\right|\left|f\left(x_{n}\right)\left\|+\left|\gamma_{n+1}-\gamma_{n}\right||| T\left(\mu_{n}\right) y_{n}\right\|\right. \\
& +\gamma_{n+1}\left\|y_{n+1}-y_{n}\right\| \\
& \leqslant\left(\alpha_{n+1}+\beta_{n+1} \psi\right)\left\|x_{n+1}-x_{n}\right\|+\gamma_{n+1} \sup _{y \in\left\{y_{n}\right\}}\left\|T\left(\mu_{n+1}\right) y-T\left(\mu_{n}\right) y\right\| \\
& +\left|\alpha_{n+1}-\alpha_{n}\right||| x_{n}||+\left|\beta_{n+1}-\beta_{n}\right||| f\left(x_{n}\right)\left\|+\left|\gamma_{n+1}-\gamma_{n}\right|\right\| T\left(\mu_{n}\right) y_{n}|| \\
& +\gamma_{n+1}\left[\left(\delta_{n+1} \psi+\left(1-\delta_{n+1}\right) \lambda_{n+1}\right)\left\|x_{n+1}-x_{n}\right\|+\left|\delta_{n+1}-\delta_{n}\right|\left(\left\|f\left(x_{n}\right)\right\|+\left\|z_{n}\right\|\right)\right. \\
& \left.+\left(1-\delta_{n+1}\right)\left|\lambda_{n+1}-\lambda_{n}\right|\left(\left\|x_{n}\right\|+\left\|x_{n+1}\right\|\right)+\left(1-\delta_{n+1}\right)\left(1-\lambda_{n+1}\right)\left\|x_{n+2}-x_{n+1}\right\|\right] \\
& =\left(\alpha_{n+1}+\beta_{n+1} \psi+\gamma_{n+1} \delta_{n+1} \psi+\gamma_{n+1}\left(1-\delta_{n+1}\right) \lambda_{n+1}\right)\left\|x_{n+1}-x_{n}\right\| \\
& +\gamma_{n+1} \sup \left\|\mathrm{T}\left(\mu_{n+1}\right) y-\mathrm{T}\left(\mu_{n}\right) y\right\|+\left|\alpha_{n+1}-\alpha_{n}\right|\left\|x_{n}\right\|+\left|\beta_{n+1}-\beta_{n}\right|\left\|f\left(x_{n}\right)\right\| \\
& y \in\left\{y_{n}\right\} \\
& +\left|\gamma_{n+1}-\gamma_{n}\right||| T\left(\mu_{n}\right) y_{n} \|+\gamma_{n+1}\left|\delta_{n+1}-\delta_{n}\right|\left(\left\|f\left(x_{n}\right)\right\|+\left\|z_{n}\right\|\right) \\
& +\gamma_{n+1}\left(1-\delta_{n+1}\right)\left|\lambda_{n+1}-\lambda_{n}\right|\left(\left\|x_{n}\right\|+\left\|x_{n+1}\right\|\right) \\
& +\gamma_{n+1}\left(1-\delta_{n+1}\right)\left(1-\lambda_{n+1}\right)\left\|x_{n+2}-x_{n+1}\right\| \\
& =\left[1-\gamma_{n+1}\left(1-\delta_{n+1}\right)\left(1-\lambda_{n+1}\right)-\left(\beta_{n+1}+\gamma_{n+1} \delta_{n+1}\right) \eta\right]\left\|x_{n+1}-x_{n}\right\| \\
& +\gamma_{n+1} \sup _{y \in\left\{y_{n}\right\}}\left\|\mathrm{T}\left(\mu_{n+1}\right) y-T\left(\mu_{n}\right) y\right\|+\left|\alpha_{n+1}-\alpha_{n}\right|\left\|x_{n}\right\|+\left|\beta_{n+1}-\beta_{n}\right|\left\|f\left(x_{n}\right)\right\| \\
& +\left|\gamma_{n+1}-\gamma_{n}\right||| \mathbf{T}\left(\mu_{n}\right) y_{n} \|+\gamma_{n+1}\left|\delta_{n+1}-\delta_{n}\right|\left(\left\|f\left(x_{n}\right)\right\|+\left\|z_{n}\right\|\right) \\
& +\gamma_{n+1}\left(1-\delta_{n+1}\right)\left|\lambda_{n+1}-\lambda_{n}\right|\left(\left\|x_{n}\right\|+\left\|x_{n+1}\right\|\right) \\
& +\gamma_{n+1}\left(1-\delta_{n+1}\right)\left(1-\lambda_{n+1}\right)\left\|x_{n+2}-x_{n+1}\right\| \text {. }
\end{aligned}
$$

\section{It follows that}

$$
\begin{aligned}
\left\|x_{n+2}-x_{n+1}\right\| \leqslant & {\left[1-\frac{\left(\beta_{n+1}+\gamma_{n+1} \delta_{n+1}\right) \eta}{1-\gamma_{n+1}\left(1-\delta_{n+1}\right)\left(1-\lambda_{n+1}\right)}\right]\left\|x_{n+1}-x_{n}\right\| } \\
& +\frac{\gamma_{n+1}}{1-\gamma_{n+1}\left(1-\delta_{n+1}\right)\left(1-\lambda_{n+1}\right)} \sup _{y \in\left\{y_{n}\right\}}\left\|T\left(\mu_{n+1}\right) y-T\left(\mu_{n}\right) y\right\| \\
& +\frac{\left|\alpha_{n+1}-\alpha_{n}\right|}{1-\gamma_{n+1}\left(1-\delta_{n+1}\right)\left(1-\lambda_{n+1}\right)}\left\|x_{n}\right\|+\frac{\left|\beta_{n+1}-\beta_{n}\right|}{1-\gamma_{n+1}\left(1-\delta_{n+1}\right)\left(1-\lambda_{n+1}\right)}\left\|f\left(x_{n}\right)\right\| \\
& +\frac{\left|\gamma_{n+1}-\gamma_{n}\right|}{1-\gamma_{n+1}\left(1-\delta_{n+1}\right)\left(1-\lambda_{n+1}\right)}\left\|T\left(\mu_{n}\right) y_{n}\right\|
\end{aligned}
$$




$$
\begin{aligned}
& +\frac{\left|\delta_{n+1}-\delta_{n}\right| \gamma_{n+1}}{1-\gamma_{n+1}\left(1-\delta_{n+1}\right)\left(1-\lambda_{n+1}\right)}\left(\left\|f\left(x_{n}\right)\right\|+\left\|z_{n}\right\|\right) \\
& +\frac{\left|\lambda_{n+1}-\lambda_{n}\right| \gamma_{n+1}\left(1-\delta_{n+1}\right)}{1-\gamma_{n+1}\left(1-\delta_{n+1}\right)\left(1-\lambda_{n+1}\right)}\left(\left\|x_{n}\right\|+\left\|x_{n+1}\right\|\right) \\
& \leqslant\left[1-\frac{\left(\beta_{n+1}+\gamma_{n+1} \delta_{n+1}\right) \eta}{1-\gamma_{n+1}\left(1-\delta_{n+1}\right)\left(1-\lambda_{n+1}\right)}\right]\left\|x_{n+1}-x_{n}\right\| \\
& +\frac{\gamma_{n+1}}{1-\gamma_{n+1}\left(1-\delta_{n+1}\right)\left(1-\lambda_{n+1}\right)} \sup _{y \in\left\{y_{n}\right\}}\left\|T\left(\mu_{n+1}\right) y-T\left(\mu_{n}\right) y\right\| \\
& +\left[\frac{\left|\alpha_{n+1}-\alpha_{n}\right|}{1-\gamma_{n+1}\left(1-\delta_{n+1}\right)\left(1-\lambda_{n+1}\right)}+\frac{\left|\beta_{n+1}-\beta_{n}\right|}{1-\gamma_{n+1}\left(1-\delta_{n+1}\right)\left(1-\lambda_{n+1}\right)}\right. \\
& +\frac{\left|\gamma_{n+1}-\gamma_{n}\right|}{1-\gamma_{n+1}\left(1-\delta_{n+1}\right)\left(1-\lambda_{n+1}\right)}+\frac{\left|\delta_{n+1}-\delta_{n}\right| \gamma_{n+1}}{1-\gamma_{n+1}\left(1-\delta_{n+1}\right)\left(1-\lambda_{n+1}\right)} \\
& \left.+\frac{\left|\lambda_{n+1}-\lambda_{n}\right| \gamma_{n+1}\left(1-\delta_{n+1}\right)}{1-\gamma_{n+1}\left(1-\delta_{n+1}\right)\left(1-\lambda_{n+1}\right)}\right] M \text {, }
\end{aligned}
$$

where $M=\sup _{n \geqslant 1}\left\{\left\|x_{n}\right\|,\left\|f\left(x_{n}\right)\right\|+\left\|T\left(\mu_{n}\right) y_{n}\right\|,\left\|f\left(x_{n}\right)\right\|+\left\|z_{n}\right\|,\left\|x_{n}\right\|+\left\|x_{n+1}\right\|\right\}<\infty$.

Setting $x_{n+1}=\left(1-\alpha_{n}\right) w_{n}+\alpha_{n} x_{n}$ for all $n \geqslant 1$, we see that $w_{n}=\frac{x_{n+1}-\alpha_{n} x_{n}}{1-\alpha_{n}}$, then we have

$$
\begin{aligned}
\left\|w_{n+1}-w_{n}\right\|= & \left\|\frac{x_{n+2}-\alpha_{n+1} x_{n+1}}{1-\alpha_{n+1}}-\frac{x_{n+1}-\alpha_{n} x_{n}}{1-\alpha_{n}}\right\| \\
= & \left\|\frac{\beta_{n+1} f\left(x_{n+1}\right)+\gamma_{n+1} T\left(\mu_{n+1}\right) y_{n+1}}{1-\alpha_{n+1}}-\frac{\beta_{n} f\left(x_{n}\right)+\gamma_{n} T\left(\mu_{n}\right) y_{n}}{1-\alpha_{n}}\right\| \\
= & \| \frac{\beta_{n+1} f\left(x_{n+1}\right)+\gamma_{n+1} T\left(\mu_{n+1}\right) y_{n+1}}{1-\alpha_{n+1}}-\frac{\beta_{n+1} f\left(x_{n}\right)}{1-\alpha_{n+1}}+\frac{\beta_{n+1} f\left(x_{n}\right)}{1-\alpha_{n+1}} \\
& -\frac{\gamma_{n+1} T\left(\mu_{n}\right) y_{n}}{1-\alpha_{n+1}}+\frac{\gamma_{n+1} T\left(\mu_{n}\right) y_{n}}{1-\alpha_{n+1}}-\frac{\beta_{n} f\left(x_{n}\right)+\gamma_{n} T\left(\mu_{n}\right) y_{n}}{1-\alpha_{n}} \| \\
= & \| \frac{\beta_{n+1}}{1-\alpha_{n+1}}\left(f\left(x_{n+1}\right)-f\left(x_{n}\right)\right)+\frac{\gamma_{n+1}}{1-\alpha_{n+1}}\left(T\left(\mu_{n+1}\right) y_{n+1}-T\left(\mu_{n}\right) y_{n}\right) \\
& +\left(\frac{\beta_{n+1}}{1-\alpha_{n+1}}-\frac{\beta_{n}}{1-\alpha_{n}}\right) f\left(x_{n}\right)+\left(\frac{\gamma_{n+1}}{1-\alpha_{n+1}}-\frac{\gamma_{n}}{1-\alpha_{n}}\right) T\left(\mu_{n}\right) y_{n} \| \\
= & \| \frac{\beta_{n+1}}{1-\alpha_{n+1}}\left(f\left(x_{n+1}\right)-f\left(x_{n}\right)\right)+\frac{\gamma_{n+1}}{1-\alpha_{n+1}}\left(T\left(\mu_{n+1}\right) y_{n+1}-T\left(\mu_{n}\right) y_{n}\right) \\
& +\left(\frac{\beta_{n+1}}{1-\alpha_{n+1}}-\frac{\beta_{n}}{1-\alpha_{n}}\right) f\left(x_{n}\right)+\left(\frac{\beta_{n}}{1-\alpha_{n}}-\frac{\beta_{n+1}}{1-\alpha_{n+1}}\right) T\left(\mu_{n}\right) y_{n} \| \\
\leqslant & \frac{\beta_{n+1}}{1-\alpha_{n+1}}\left\|f\left(x_{n+1}\right)-f\left(x_{n}\right)\right\|+\frac{\gamma_{n+1}}{1-\alpha_{n+1}}\left\|T\left(\mu_{n+1}\right) y_{n+1}-T\left(\mu_{n}\right) y_{n}\right\| \\
& +\left|\frac{\beta_{n+1}}{1-\alpha_{n+1}}-\frac{\beta_{n}}{1-\alpha_{n}}\right|\left(\left\|f\left(x_{n}\right)\right\|+\left\|T\left(\mu_{n}\right) y_{n}\right\|\right) \\
\leqslant & \frac{\beta_{n+1} \psi}{1-\alpha_{n+1}}\left\|x_{n+1}-x_{n}\right\|+\left|\frac{\beta_{n+1}}{1-\alpha_{n+1}}-\frac{\beta_{n}}{1-\alpha_{n}}\right|\left(\left\|f\left(x_{n}\right)\right\|+\left\|T\left(\mu_{n}\right) y_{n}\right\|\right) \\
& +\frac{\gamma_{n+1}}{1-\alpha_{n+1}}\left(\left\|T\left(\mu_{n+1}\right) y_{n+1}-T\left(\mu_{n+1}\right) y_{n}\right\|+\left\|T\left(\mu_{n+1}\right) y_{n}-T\left(\mu_{n}\right) y_{n}\right\|\right) \\
\leqslant & \frac{\beta_{n+1} \psi}{1-\alpha_{n+1}}\left\|x_{n+1}-x_{n}\right\|+\left|\frac{\beta_{n+1}}{1-\alpha_{n+1}}-\frac{\beta_{n}}{1-\alpha_{n}}\right|\left(\left\|f\left(x_{n}\right)\right\|+\left\|T\left(\mu_{n}\right) y_{n}\right\|\right) \\
&
\end{aligned}
$$




$$
\begin{aligned}
& +\frac{\gamma_{n+1}}{1-\alpha_{n+1}}\left\|y_{n+1}-y_{n}\right\|+\frac{\gamma_{n+1}}{1-\alpha_{n+1}} \sup _{y \in\left\{y_{n}\right\}}\left\|T\left(\mu_{n+1}\right) y-T\left(\mu_{n}\right) y\right\| \\
& \leqslant \frac{\beta_{n+1} \psi}{1-\alpha_{n+1}}\left\|x_{n+1}-x_{n}\right\|+\frac{\gamma_{n+1}}{1-\alpha_{n+1}} \sup _{y \in\left\{y_{n}\right\}}\left\|T\left(\mu_{n+1}\right) y-T\left(\mu_{n}\right) y\right\| \\
& +\left|\frac{\beta_{n+1}}{1-\alpha_{n+1}}-\frac{\beta_{n}}{1-\alpha_{n}}\right|\left(\left\|f\left(x_{n}\right)\right\|+\left\|T\left(\mu_{n}\right) y_{n}\right\|\right) \\
& +\frac{\gamma_{n+1}}{1-\alpha_{n+1}}\left[\left(\delta_{n+1} \psi+\left(1-\delta_{n+1}\right) \lambda_{n+1}\right)\left\|x_{n+1}-x_{n}\right\|+\left|\delta_{n+1}-\delta_{n}\right|\left(\left\|f\left(x_{n}\right)\right\|+\left\|z_{n}\right\|\right)\right. \\
& \left.+\left(1-\delta_{n+1}\right)\left|\lambda_{n+1}-\lambda_{n}\right|\left(\left\|x_{n}\right\|+\left\|x_{n+1}\right\|\right)+\left(1-\delta_{n+1}\right)\left(1-\lambda_{n+1}\right)\left\|x_{n+2}-x_{n+1}\right\|\right] \\
& \leqslant \frac{1}{1-\alpha_{n+1}}\left[\beta_{n+1} \psi+\gamma_{n+1} \delta_{n+1} \psi+\gamma_{n+1}\left(1-\delta_{n+1}\right) \lambda_{n+1}\right]\left\|x_{n+1}-x_{n}\right\| \\
& +\frac{\gamma_{n+1}}{1-\alpha_{n+1}} \sup _{y \in\left\{y_{n}\right\}}\left\|T\left(\mu_{n+1}\right) y-T\left(\mu_{n}\right) y\right\|+\left|\frac{\beta_{n+1}}{1-\alpha_{n+1}}-\frac{\beta_{n}}{1-\alpha_{n}}\right|\left(\left\|f\left(x_{n}\right)\right\|+\left\|T\left(\mu_{n}\right) y_{n}\right\|\right) \\
& +\frac{\gamma_{n+1}}{1-\alpha_{n+1}}\left|\delta_{n+1}-\delta_{n}\right|\left(\left\|f\left(x_{n}\right)\right\|+\left\|z_{n}\right\|\right)+\frac{\gamma_{n+1}}{1-\alpha_{n+1}}\left(1-\delta_{n+1}\right)\left|\lambda_{n+1}-\lambda_{n}\right|\left(\left\|x_{n}\right\|+\left\|x_{n+1}\right\|\right) \\
& +\frac{\gamma_{n+1}}{1-\alpha_{n+1}}\left(1-\delta_{n+1}\right)\left(1-\lambda_{n+1}\right)\left\|x_{n+2}-x_{n+1}\right\| \\
& \leqslant \frac{1}{1-\alpha_{n+1}}\left[\beta_{n+1} \psi+\gamma_{n+1} \delta_{n+1} \psi+\gamma_{n+1}\left(1-\delta_{n+1}\right) \lambda_{n+1}\right]\left\|x_{n+1}-x_{n}\right\| \\
& +\frac{\gamma_{n+1}}{1-\alpha_{n+1}} \sup _{y \in\left\{y_{n}\right\}}\left\|\mathrm{T}\left(\mu_{n+1}\right) y-T\left(\mu_{n}\right) y\right\|+\left|\frac{\beta_{n+1}}{1-\alpha_{n+1}}-\frac{\beta_{n}}{1-\alpha_{n}}\right|\left(\left\|f\left(x_{n}\right)\right\|+\left\|T\left(\mu_{n}\right) y_{n}\right\|\right) \\
& +\frac{\gamma_{n+1}}{1-\alpha_{n+1}}\left|\delta_{n+1}-\delta_{n}\right|\left(\left\|f\left(x_{n}\right)\right\|+\left\|z_{n}\right\|\right)+\frac{\gamma_{n+1}}{1-\alpha_{n+1}}\left(1-\delta_{n+1}\right)\left|\lambda_{n+1}-\lambda_{n}\right|\left(\left\|x_{n}\right\|+\left\|x_{n+1}\right\|\right) \\
& +\frac{\gamma_{n+1}}{1-\alpha_{n+1}}\left(1-\delta_{n+1}\right)\left(1-\lambda_{n+1}\right)\left\{\left[1-\frac{\left(\beta_{n+1}+\gamma_{n+1} \delta_{n+1}\right) \eta}{1-\gamma_{n+1}\left(1-\delta_{n+1}\right)\left(1-\lambda_{n+1}\right)}\right]\left\|x_{n+1}-x_{n}\right\|\right. \\
& +\frac{\gamma_{n+1}}{1-\gamma_{n+1}\left(1-\delta_{n+1}\right)\left(1-\lambda_{n+1}\right)} \sup _{y \in\left\{y_{n}\right\}}\left\|T\left(\mu_{n+1}\right) y-T\left(\mu_{n}\right) y\right\| \\
& +\left[\frac{\left|\alpha_{n+1}-\alpha_{n}\right|}{1-\gamma_{n+1}\left(1-\delta_{n+1}\right)\left(1-\lambda_{n+1}\right)}+\frac{\left|\beta_{n+1}-\beta_{n}\right|}{1-\gamma_{n+1}\left(1-\delta_{n+1}\right)\left(1-\lambda_{n+1}\right)}\right. \\
& +\frac{\left|\gamma_{n+1}-\gamma_{n}\right|}{1-\gamma_{n+1}\left(1-\delta_{n+1}\right)\left(1-\lambda_{n+1}\right)}+\frac{\left|\delta_{n+1}-\delta_{n}\right| \gamma_{n+1}}{1-\gamma_{n+1}\left(1-\delta_{n+1}\right)\left(1-\lambda_{n+1}\right)} \\
& \left.\left.+\frac{\left|\lambda_{n+1}-\lambda_{n}\right| \gamma_{n+1}\left(1-\delta_{n+1}\right)}{1-\gamma_{n+1}\left(1-\delta_{n+1}\right)\left(1-\lambda_{n+1}\right)}\right] M\right\} \\
& \leqslant \frac{1}{1-\alpha_{n+1}}\left[\beta_{n+1} \psi+\gamma_{n+1} \delta_{n+1} \psi+\gamma_{n+1}\left(1-\delta_{n+1}\right) \lambda_{n+1}\right. \\
& \left.+\gamma_{n+1}\left(1-\delta_{n+1}\right)\left(1-\lambda_{n+1}\right)\right]\left\|x_{n+1}-x_{n}\right\| \\
& +\frac{\gamma_{n+1}}{1-\alpha_{n+1}} \sup _{y \in\left\{y_{n}\right\}}\left\|\mathrm{T}\left(\mu_{n+1}\right) y-T\left(\mu_{n}\right) y\right\|+\left|\frac{\beta_{n+1}}{1-\alpha_{n+1}}-\frac{\beta_{n}}{1-\alpha_{n}}\right|\left(\left\|f\left(x_{n}\right)\right\|+\left\|T\left(\mu_{n}\right) y_{n}\right\|\right) \\
& +\frac{\gamma_{n+1}}{1-\alpha_{n+1}}\left|\delta_{n+1}-\delta_{n}\right|\left(\left\|f\left(x_{n}\right)\right\|+\left\|z_{n}\right\|\right)+\frac{\gamma_{n+1}}{1-\alpha_{n+1}}\left(1-\delta_{n+1}\right)\left|\lambda_{n+1}-\lambda_{n}\right|\left(\left\|x_{n}\right\|+\left\|x_{n+1}\right\|\right) \\
& +\frac{\gamma_{n+1}}{1-\alpha_{n+1}}\left(1-\delta_{n+1}\right)\left(1-\lambda_{n+1}\right) \\
& \times\left\{\frac{\gamma_{n+1}}{1-\gamma_{n+1}\left(1-\delta_{n+1}\right)\left(1-\lambda_{n+1}\right)} \sup _{y \in\left\{y_{n}\right\}}\left\|T\left(\mu_{n+1}\right) y-T\left(\mu_{n}\right) y\right\|\right.
\end{aligned}
$$




$$
\begin{aligned}
& +\left[\frac{\left|\alpha_{n+1}-\alpha_{n}\right|}{1-\gamma_{n+1}\left(1-\delta_{n+1}\right)\left(1-\lambda_{n+1}\right)}+\frac{\left|\beta_{n+1}-\beta_{n}\right|}{1-\gamma_{n+1}\left(1-\delta_{n+1}\right)\left(1-\lambda_{n+1}\right)}\right. \\
& +\frac{\left|\gamma_{n+1}-\gamma_{n}\right|}{1-\gamma_{n+1}\left(1-\delta_{n+1}\right)\left(1-\lambda_{n+1}\right)}+\frac{\left|\delta_{n+1}-\delta_{n}\right| \gamma_{n+1}}{1-\gamma_{n+1}\left(1-\delta_{n+1}\right)\left(1-\lambda_{n+1}\right)} \\
& \left.\left.+\frac{\left|\lambda_{n+1}-\lambda_{n}\right| \gamma_{n+1}\left(1-\delta_{n+1}\right)}{1-\gamma_{n+1}\left(1-\delta_{n+1}\right)\left(1-\lambda_{n+1}\right)}\right] M\right\} \\
& =\left[1-\frac{\left(\beta_{n+1}+\gamma_{n+1} \delta_{n+1}\right) \eta}{1-\alpha_{n+1}}\right]\left\|x_{n+1}-x_{n}\right\| \\
& +\frac{\gamma_{n+1}}{1-\alpha_{n+1}} \sup _{y \in\left\{y_{n}\right\}}\left\|T\left(\mu_{n+1}\right) y-T\left(\mu_{n}\right) y\right\|+\left|\frac{\beta_{n+1}}{1-\alpha_{n+1}}-\frac{\beta_{n}}{1-\alpha_{n}}\right|\left(\left\|f\left(x_{n}\right)\right\|+\left\|T\left(\mu_{n}\right) y_{n}\right\|\right) \\
& +\frac{\gamma_{n+1}}{1-\alpha_{n+1}}\left|\delta_{n+1}-\delta_{n}\right|\left(\left\|f\left(x_{n}\right)\right\|+\left\|z_{n}\right\|\right)+\frac{\gamma_{n+1}}{1-\alpha_{n+1}}\left(1-\delta_{n+1}\right)\left|\lambda_{n+1}-\lambda_{n}\right|\left(\left\|x_{n}\right\|+\left\|x_{n+1}\right\|\right) \\
& +\frac{\gamma_{n+1}}{1-\alpha_{n+1}}\left(1-\delta_{n+1}\right)\left(1-\lambda_{n+1}\right) \\
& +\left\{\frac{\gamma_{n+1}}{1-\gamma_{n+1}\left(1-\delta_{n+1}\right)\left(1-\lambda_{n+1}\right)} \sup _{y \in\left\{y_{n}\right\}}|| T\left(\mu_{n+1}\right) y-T\left(\mu_{n}\right) y \|\right. \\
& +\left[\frac{\left|\alpha_{n+1}-\alpha_{n}\right|}{1-\gamma_{n+1}\left(1-\delta_{n+1}\right)\left(1-\lambda_{n+1}\right)}+\frac{\left|\beta_{n+1}-\beta_{n}\right|}{1-\gamma_{n+1}\left(1-\delta_{n+1}\right)\left(1-\lambda_{n+1}\right)}\right. \\
& +\frac{\left|\gamma_{n+1}-\gamma_{n}\right|}{1-\gamma_{n+1}\left(1-\delta_{n+1}\right)\left(1-\lambda_{n+1}\right)}+\frac{\left|\delta_{n+1}-\delta_{n}\right| \gamma_{n+1}}{1-\gamma_{n+1}\left(1-\delta_{n+1}\right)\left(1-\lambda_{n+1}\right)} \\
& \left.+\frac{\left|\lambda_{n+1}-\lambda_{n}\right| \gamma_{n+1}\left(1-\delta_{n+1}\right)}{1-\gamma_{n+1}\left(1-\delta_{n+1}\right)\left(1-\lambda_{n+1}\right)} M\right\} . \\
&
\end{aligned}
$$

Therefore,

$$
\limsup _{n \rightarrow \infty}\left(\left\|w_{n+1}-w_{n}\right\|-\left\|x_{n+1}-x_{n}\right\|\right) \leqslant 0 .
$$

Applying Lemma 2.1, we obtain $\lim _{n \rightarrow \infty}\left\|w_{n}-x_{n}\right\|=0$ and by setting $\left\{w_{n}\right\}$, we also have

$$
\lim _{n \rightarrow \infty}\left\|x_{n+1}-x_{n}\right\|=0
$$

Next, we will show that $\lim _{n \rightarrow \infty}\left\|x_{n}-T\left(\mu_{n}\right) x_{n}\right\|=0$. We observe that

$$
\left\|z_{n}-x_{n}\right\|=\left(1-\lambda_{n}\right)\left\|x_{n+1}-x_{n}\right\| \text { and }\left\|y_{n}-z_{n}\right\|=\delta_{n} \| f\left(x_{)}-z_{n} \|,\right.
$$

which imply that

$$
\lim _{n \rightarrow \infty}\left\|z_{n}-x_{n}\right\|=0 \text { and } \lim _{n \rightarrow \infty}\left\|y_{n}-z_{n}\right\|=0 .
$$

Therefore, we conclude that

$$
\lim _{n \rightarrow \infty}\left\|y_{n}-x_{n}\right\|=0
$$

Consider that

$$
\begin{aligned}
\left\|x_{n}-T\left(\mu_{n}\right) x_{n}\right\| & \leqslant\left\|x_{n}-x_{n+1}\right\|+\left\|x_{n+1}-T\left(\mu_{n}\right) x_{n}\right\| \\
& =\left\|x_{n}-x_{n+1}\right\|+\left\|\alpha_{n} x_{n}+\beta_{n} f\left(x_{n}\right)+\gamma_{n} T\left(\mu_{n}\right) y_{n}-T\left(\mu_{n}\right) x_{n}\right\| \\
& \leqslant\left\|x_{n}-x_{n+1}\right\|+\alpha_{n}\left\|x_{n}-T\left(\mu_{n}\right) x_{n}\right\|+\beta_{n}\left\|f\left(x_{n}\right)-T\left(\mu_{n}\right) x_{n}\right\|+\gamma_{n}\left\|T\left(\mu_{n}\right) y_{n}-T\left(\mu_{n}\right) x_{n}\right\| \\
& \leqslant\left\|x_{n}-x_{n+1}\right\|+\alpha_{n}\left\|x_{n}-T\left(\mu_{n}\right) x_{n}\right\|+\beta_{n}\left\|f\left(x_{n}\right)-T\left(\mu_{n}\right) x_{n}\right\|+\gamma_{n}\left\|y_{n}-x_{n}\right\|
\end{aligned}
$$


which implies that

$$
\left\|x_{n}-T\left(\mu_{n}\right) x_{n}\right\| \leqslant \frac{1}{1-\alpha_{n}}\left\|x_{n}-x_{n+1}\right\|+\frac{\beta_{n}}{1-\alpha_{n}}\left\|f\left(x_{n}\right)-T\left(\mu_{n}\right) x_{n}\right\|+\frac{\gamma_{n}}{1-\alpha_{n}}\left\|y_{n}-x_{n}\right\| .
$$

It follows from the conditions (ii), (iii), and (3.2) that

$$
\lim _{n \rightarrow \infty}\left\|x_{n}-T\left(\mu_{n}\right) x_{n}\right\|=0 .
$$

Now, we show that $z \in F(\mathcal{S})$. We can choose a sequence $\left\{x_{n_{k}}\right\}$ of $\left\{x_{n}\right\}$ such that $\left\{x_{n_{k}}\right\}$ is bounded and there exists a subsequence $\left\{x_{n_{k_{j}}}\right\}$ of $\left\{x_{n_{k}}\right\}$ which converges weakly to $z$. Without loss of generality, we can assume that $x_{n_{k}} \rightarrow z$. Let $\mu_{n_{k}} \geqslant 0$ such that $\mu_{n_{k}} \rightarrow 0$ and $\frac{\left\|T\left(\mu_{n_{k}}\right) x_{n_{k}}-x_{n_{k}}\right\|}{\mu_{n_{k}}} \rightarrow 0, k \rightarrow \infty$. Fix $t>0$, we can notice that

$$
\begin{aligned}
\left\|x_{n_{k}}-T(t) z\right\| \leqslant & \sum_{i=0}^{\left[t / \mu_{n_{k}}\right]-1}\left\|t\left((i+1) \mu_{n_{k}}\right) x_{n_{k}}-T\left(i \mu_{n_{k}}\right) x_{n_{k}}\right\| \\
& +\left\|T\left(\left[t / \mu_{n_{k}}\right] \mu_{k}\right) x_{n_{k}}-T\left(\left[t / \mu_{n_{k}}\right] \mu_{n_{k}}\right) z\right\|+\left\|T\left(\left[t / \mu_{n_{k}}\right] \mu_{n_{k}}\right) z-T(t) z\right\| \\
\leqslant & {\left[t / \mu_{n_{k}}\right]\left\|T\left(\mu_{n_{k}}\right) x_{n_{k}}-x_{n_{k}}\right\|+\left\|x_{n_{k}}-z\right\|+\left\|T\left(t-\left[t / \mu_{n_{k}}\right] \mu_{n_{k}}\right) z-z\right\| } \\
\leqslant & t \frac{\left\|T\left(\mu_{n_{k}}\right) x_{n_{k}}-x_{n_{k}}\right\|}{\mu_{n_{k}}}+\left\|x_{n_{k}}-z\right\|+\left\|T\left(t-\left[t / \mu_{n_{k}}\right] \mu_{n_{k}}\right) z-z\right\| \\
\leqslant & t \frac{\left\|T\left(\mu_{n_{k}}\right) x_{n_{k}}-x_{n_{k}}\right\|}{\mu_{n_{k}}}+\left\|x_{n_{k}}-z\right\|+\max \left\{\|T(\mu) z-z\|: 0 \leqslant \mu \leqslant \mu_{n_{k}}\right\} .
\end{aligned}
$$

For all $k \in \mathbb{N}$, we have

$$
\limsup _{k \rightarrow \infty}\left\|x_{n_{k}}-T(t) z\right\| \leqslant \limsup _{k \rightarrow \infty}\left\|x_{n_{k}}-z\right\| .
$$

Since a Banach space $E$ with a weakly sequentially continuous duality mapping satisfies the Opial's condition, this implies $\mathrm{T}(\mathrm{t}) z=z$. Therefore, $z \in \mathrm{F}(\mathcal{S})$.

By Lemma 2.2, the sequence $\left\{x_{t}\right\}$ is defined by $x_{t}=\operatorname{tf}\left(x_{t}\right)+(1-t) T x_{t}$ such that $t \in(0,1)$ which converges strongly to a fixed point $x^{*} \in F(\mathcal{S})$ and solves the variational inequality:

$$
\left\langle(\mathrm{f}-\mathrm{I}) \chi^{*}, \mathrm{~J}\left(z-x^{*}\right)\right\rangle \leqslant 0, \forall z \in \mathrm{F}(\mathcal{S}) .
$$

Following Lemma 2.3 and (3.1)-(3.3), we conclude that

$$
\limsup _{n \rightarrow \infty}\left\langle(f-I) x^{*}, J\left(y_{n}-x^{*}\right)\right\rangle \leqslant 0
$$

and

$$
\limsup _{n \rightarrow \infty}\left\langle(f-I) x^{*}, J\left(x_{n+1}-x^{*}\right)\right\rangle \leqslant 0 .
$$

Finally, we show that $\left\{x_{n}\right\}$ converges strongly to $x^{*} \in F(\mathcal{S})$. Assume that the sequence $\left\{x_{n}\right\}$ does not converge strongly to $x^{*}$, there exists a subsequence $\left\{x_{n_{k}}\right\}$ of $\left\{x_{n}\right\}$ and $\epsilon>0$ such that $\left\|x_{n_{k}}-x^{*}\right\| \geqslant \epsilon, \forall k=$ $1,2, \ldots$. For this $\epsilon$ there exists $r \in(0,1)$ such that

$$
\left\|f\left(x_{n_{k}}\right)-f\left(x^{*}\right)\right\| \leqslant r\left\|x_{n_{k}}-x^{*}\right\| .
$$

We compute that

$$
\begin{aligned}
\left\|y_{n_{k}}-x^{*}\right\|^{2} & =\left\langle y_{n_{k}}-x^{*}, J\left(y_{n_{k}}-x^{*}\right)\right\rangle \\
& =\left\langle\delta_{n_{k}} f\left(x_{n_{k}}\right)+\left(1-\delta_{n_{k}}\right) z_{n_{k}}-x^{*}, J\left(y_{n_{k}}-x^{*}\right)\right\rangle
\end{aligned}
$$




$$
\begin{aligned}
= & \left\langle\delta_{n_{k}}\left(f\left(x_{n_{k}}\right)-x^{*}\right)+\left(1-\delta_{n_{k}}\right)\left(z_{n_{k}}-x^{*}\right), J\left(y_{n_{k}}-x^{*}\right)\right\rangle \\
= & \delta_{n_{k}}\left\langle f\left(x_{n_{k}}\right)-f\left(x^{*}\right), J\left(y_{n_{k}}-x^{*}\right)\right\rangle+\delta_{n_{k}}\left\langle f\left(x^{*}\right)-x^{*}, J\left(y_{n_{k}}-x^{*}\right)\right\rangle \\
& +\left(1-\delta_{n_{k}}\right)\left\langle z_{n_{k}}-x^{*}, J\left(y_{n_{k}}-x^{*}\right)\right\rangle \\
= & \delta_{n_{k}}\left\langle f\left(x_{n_{k}}\right)-f\left(x^{*}\right), J\left(y_{n_{k}}-x^{*}\right)\right\rangle+\delta_{n_{k}}\left\langle f\left(x^{*}\right)-x^{*}, J\left(y_{n_{k}}-x^{*}\right)\right\rangle \\
& +\left(1-\delta_{n_{k}}\right)\left\langle\lambda_{n_{k}} x_{n_{k}}+\left(1-\lambda_{n_{k}}\right) x_{n_{k+1}}-x^{*}, J\left(y_{n_{k}}-x^{*}\right)\right\rangle \\
= & \delta_{n_{k}}\left\langle f\left(x_{n_{k}}\right)-f\left(x^{*}\right), J\left(y_{n_{k}}-x^{*}\right)\right\rangle+\delta_{n_{k}}\left\langle f\left(x^{*}\right)-x^{*}, J\left(y_{n_{k}}-x^{*}\right)\right\rangle \\
& +\left(1-\delta_{n_{k}}\right)\left\langle\lambda_{n_{k}}\left(x_{n_{k}}-x^{*}\right)+\left(1-\lambda_{n_{k}}\right)\left(x_{n_{k+1}}-x^{*}\right), J\left(y_{n_{k}}-x^{*}\right)\right\rangle \\
= & \delta_{n_{k}}\left\langle f\left(x_{n_{k}}\right)-f\left(x^{*}\right), J\left(y_{n_{k}}-x^{*}\right)\right\rangle+\delta_{n_{k}}\left\langle f\left(x^{*}\right)-x^{*}, J\left(y_{n_{k}}-x^{*}\right)\right\rangle \\
& +\left(1-\delta_{n_{k}}\right) \lambda_{n_{k}}\left\langle x_{n_{k}}-x^{*}, J\left(y_{n_{k}}-x^{*}\right)\right\rangle+\left(1-\delta_{n_{k}}\right)\left(1-\lambda_{n_{k}}\right)\left\langle x_{n_{k+1}}-x^{*}, J\left(y_{n_{k}}-x^{*}\right)\right\rangle \\
\leqslant & r \delta_{n_{k}}\left\|x_{n_{k}}-x^{*}\right\|\left\|y_{n_{k}}-x^{*}\right\|+\delta_{n_{k}}\left\langle f\left(x^{*}\right)-x^{*}, J\left(y_{n_{k}}-x^{*}\right)\right\rangle \\
& +\left(1-\delta_{n_{k}}\right) \lambda_{n_{k}}\left\|x_{n_{k}}-x^{*}\right\|\left\|y_{n_{k}}-x^{*}\right\|+\left(1-\delta_{n_{k}}\right)\left(1-\lambda_{n_{k}}\right)\left\|y_{n_{k}}-x^{*}\right\|\left\|x_{n_{k+1}}-x^{*}\right\| \\
\leqslant & {\left[r \delta_{n_{k}}+\left(1-\delta_{n_{k}}\right) \lambda_{n_{k}}\right] \frac{1}{2}\left(\left\|x_{n_{k}}-x^{*}\right\|^{2}+\left\|y_{n_{k}}-x^{*}\right\|^{2}\right)+\delta_{n_{k}}\left\langle f\left(x^{*}\right)-x^{*}, J\left(y_{n_{k}}-x^{*}\right)\right\rangle } \\
& +\left(1-\delta_{n_{k}}\right)\left(1-\lambda_{n_{k}}\right) \frac{1}{2}\left(\left\|y_{n_{k}}-x^{*}\right\|^{2}+\left\|x_{n_{k+1}}-x^{*}\right\|^{2}\right) \\
= & \frac{r \delta_{n_{k}}+\left(1-\delta_{n_{k}}\right) \lambda_{n_{k}}}{2} \\
& +\delta_{n_{k}}\left\langlef \left( x_{n_{k}}-x^{*}\left\|^{2}+\frac{1-x_{n_{k}}(1-r)}{2}\right\| y_{n_{k}}-x^{*} \|^{2}\right.\right. \\
& \left.\left(y_{n_{k}}-x^{*}\right)\right\rangle+\frac{\left(1-\delta_{n_{k}}\right)\left(1-\lambda_{n_{k}}\right)}{2}\left\|x_{n_{k+1}}-x^{*}\right\|^{2},
\end{aligned}
$$

which implies that

$$
\begin{aligned}
\left\|y_{n_{k}}-x^{*}\right\|^{2} \leqslant & \frac{r \delta_{n_{k}}+\left(1-\delta_{n_{k}}\right) \lambda_{n_{k}}}{1+\delta_{n_{k}}(1-r)}\left\|x_{n_{k}}-x^{*}\right\|^{2}+\frac{2 \delta_{n_{k}}}{1+\delta_{n_{k}}(1-r)}\left\langle f\left(x^{*}\right)-x^{*}, J\left(y_{n_{k}}-x^{*}\right)\right\rangle \\
& +\frac{\left(1-\delta_{n_{k}}\right)\left(1-\lambda_{n_{k}}\right)}{1+\delta_{n_{k}}(1-r)}\left\|x_{n_{k+1}}-x^{*}\right\|^{2} .
\end{aligned}
$$

We observe that

$$
\begin{aligned}
\left\|x_{n_{k+1}}-x^{*}\right\|^{2}= & \left\langle x_{n_{k+1}}-x^{*}, J\left(x_{n_{k+1}}-x^{*}\right)\right\rangle \\
= & \left\langle\alpha_{n_{k}} x_{n_{k}}+\beta_{n_{k}} f\left(x_{n_{k}}\right)+\gamma_{n_{k}} T\left(\mu_{n_{k}}\right) y_{n_{k}}-x^{*}, J\left(x_{n_{k+1}}-x^{*}\right)\right\rangle \\
= & \left\langle\alpha_{n_{k}}\left(x_{n_{k}}-x^{*}\right)+\beta_{n_{k}}\left(f\left(x_{n_{k}}\right)-x^{*}\right)+\gamma_{n_{k}}\left(T\left(\mu_{n_{k}}\right) y_{n_{k}}-x^{*}\right), J\left(x_{n_{k+1}}-x^{*}\right)\right\rangle \\
= & \alpha_{n_{k}}\left\langle x_{n_{k}}-x^{*}, J\left(x_{n_{k+1}}-x^{*}\right)\right\rangle+\beta_{n_{k}}\left\langle f\left(x_{n_{k}}\right)-f\left(x^{*}\right), J\left(x_{n_{k+1}}-x^{*}\right)\right\rangle \\
& +\beta_{n_{k}}\left\langle f\left(x^{*}\right)-x^{*}, J\left(x_{n_{k+1}}-x^{*}\right)\right\rangle+\gamma_{n_{k}}\left\langle T\left(\mu_{n_{k}}\right) y_{n_{k}}-x^{*}, J\left(x_{n_{k+1}}-x^{*}\right)\right\rangle \\
\leqslant & \alpha_{n_{k}}\left\|x_{n_{k}}-x^{*}\right\|\left\|x_{n_{k+1}}-x^{*}\right\|+r \beta_{n_{k}}\left\|x_{n_{k}}-x^{*}\right\|\left\|x_{n_{k+1}}-x^{*}\right\| \\
& +\beta_{n_{k}}\left\langle f\left(x^{*}\right)-x^{*}, J\left(x_{n_{k+1}}-x^{*}\right)\right\rangle+\gamma_{n_{k}}\left\|y_{n_{k}}-x^{*}\right\|\left\|x_{n_{k+1}}-x^{*}\right\| \\
\leqslant & \left(\alpha_{n_{k}}+r \beta_{n_{k}}\right) \frac{1}{2}\left(\left\|x_{n_{k}}-x^{*}\right\|^{2}+\left\|x_{n_{k+1}}-x^{*}\right\|^{2}\right)+\beta_{n_{k}}\left\langle f\left(x^{*}\right)-x^{*}, J\left(x_{n_{k+1}}-x^{*}\right)\right\rangle \\
& +\gamma_{n_{k}} \frac{1}{2}\left(\left\|y_{n_{k}}-x^{*}\right\|^{2}+\left\|x_{n_{k+1}}-x^{*}\right\|^{2}\right) \\
= & \frac{\alpha_{n_{k}}+r \beta_{n_{k}}}{2}\left\|x_{n_{k}}-x^{*}\right\|^{2}+\frac{\alpha_{n_{k}}+r \beta_{n_{k}}}{2}\left\|x_{n_{k+1}}-x^{*}\right\|^{2} \\
& +\beta_{n_{k}}\left\langle f\left(x^{*}\right)-x^{*}, J\left(x_{n_{k+1}}-x^{*}\right)\right\rangle+\frac{\gamma_{n_{k}}}{2}\left\|y_{n_{k}}-x^{*}\right\|^{2}+\frac{\gamma_{n_{k}}}{2}\left\|x_{n_{k+1}}-x^{*}\right\|^{2} \\
\leqslant & \frac{\alpha_{n_{k}}+r \beta_{n_{k}}}{2}\left\|x_{n_{k}}-x^{*}\right\|^{2}+\frac{\alpha_{n_{k}}+r \beta_{n_{k}}}{2}\left\|x_{n_{k+1}}-x^{*}\right\|^{2}+\beta_{n_{k}}\left\langle f\left(x^{*}\right)-x^{*}, J\left(x_{n_{k+1}}-x^{*}\right)\right\rangle \\
& +\frac{\gamma_{n_{k}}}{2}\left\|x_{n_{k+1}}-x^{*}\right\|^{2}+\frac{\gamma_{n_{k}}}{2}\left[\frac{r \delta_{n_{k}}+\left(1-\delta_{n_{k}}\right) \lambda_{n_{k}}}{1+\delta_{n_{k}}(1-r)}\left\|x_{n_{k}}-x^{*}\right\|^{2}\right.
\end{aligned}
$$




$$
\begin{aligned}
& \left.+\frac{2 \delta_{n_{k}}}{1+\delta_{n_{k}}(1-r)}\left\langle f\left(x^{*}\right)-x^{*}, J\left(y_{n_{k}}-x^{*}\right)\right\rangle+\frac{\left(1-\delta_{n_{k}}\right)\left(1-\lambda_{n_{k}}\right)}{1+\delta_{n_{k}}(1-r)}\left\|x_{n_{k+1}}-x^{*}\right\|^{2}\right] \\
& =\left[\frac{\alpha_{n_{k}}+r \beta_{n_{k}}}{2}+\frac{\gamma_{n_{k}}}{2}\left(\frac{r \delta_{n_{k}}+\left(1-\delta_{n_{k}}\right) \lambda_{n_{k}}}{1+\delta_{n_{k}}(1-r)}\right)\right]\left\|x_{n_{k}}-x^{*}\right\|^{2} \\
& +\left[\frac{\alpha_{n_{k}}+r \beta_{n_{k}}}{2}+\frac{\gamma_{n_{k}}}{2}+\frac{\gamma_{n_{k}}}{2}\left(\frac{\left(1-\delta_{n_{k}}\right)\left(1-\lambda_{n_{k}}\right)}{1+\delta_{n_{k}}(1-r)}\right)\right]\left\|x_{n_{k+1}}-x^{*}\right\|^{2} \\
& +\beta_{n_{k}}\left\langle f\left(x^{*}\right)-x^{*}, J\left(x_{n_{k+1}}-x^{*}\right)\right\rangle+\frac{\gamma_{n_{k}} \delta_{n_{k}}}{1+\delta_{n_{k}}(1-r)}\left\langle f\left(x^{*}\right)-x^{*}, J\left(y_{n_{k}}-x^{*}\right)\right\rangle \\
& =\frac{\left(1-\gamma_{n_{k}}-\beta_{n_{k}}(1-r)\right)\left(1+\delta_{n_{k}}(1-r)\right)+\gamma_{n_{k}}\left(r \delta_{n_{k}}+\left(1-\delta_{n_{k}}\right) \lambda_{n_{k}}\right)}{2\left(1+\delta_{n_{k}}(1-r)\right)}\left\|x_{n_{k}}-x^{*}\right\|^{2} \\
& +\frac{\left(1-\beta_{n_{k}}(1-r)\right)\left(1+\delta_{n_{k}}(1-r)\right)+\gamma_{n_{k}}\left(1-\delta_{n_{k}}\right)\left(1-\lambda_{n_{k}}\right)}{2\left(1+\delta_{n_{k}}(1-r)\right)}\left\|x_{n_{k+1}}-x^{*}\right\|^{2} \\
& +\beta_{n_{k}}\left\langle f\left(x^{*}\right)-x^{*}, J\left(x_{n_{k+1}}-x^{*}\right)\right\rangle+\frac{\gamma_{n_{k}} \delta_{n_{k}}}{1+\delta_{n_{k}}(1-r)}\left\langle f\left(x^{*}\right)-x^{*}, J\left(y_{n_{k}}-x^{*}\right)\right\rangle .
\end{aligned}
$$

\section{It follows that}

$$
\begin{aligned}
\left\|x_{n_{k+1}}-x^{*}\right\|^{2} \leqslant & \frac{\left(1-\gamma_{n_{k}}-\beta_{n_{k}}(1-r)\right)\left(1+\delta_{n_{k}}(1-r)\right)+\gamma_{n_{k}}\left(r \delta_{n_{k}}+\left(1-\delta_{n_{k}}\right) \lambda_{n_{k}}\right)}{\left(1+\beta_{n_{k}}(1-r)\right)\left(1+\delta_{n_{k}}(1-r)\right)-\gamma_{n_{k}}\left(1-\delta_{n_{k}}\right)\left(1-\lambda_{n_{k}}\right)}\left\|x_{n_{k}}-x^{*}\right\|^{2} \\
& +\frac{2 \beta_{n_{k}}\left(1+\delta_{n_{k}}(1-r)\right)}{\left(1+\beta_{n_{k}}(1-r)\right)\left(1+\delta_{n_{k}}(1-r)\right)-\gamma_{n_{k}}\left(1-\delta_{n_{k}}\right)\left(1-\lambda_{n_{k}}\right)}\left\langle f\left(x^{*}\right)-x^{*}, J\left(x_{n_{k+1}}-x^{*}\right)\right\rangle \\
& +\frac{2 \gamma_{n_{k}} \delta_{n_{k}}}{\left(1+\beta_{n_{k}}(1-r)\right)\left(1+\delta_{n_{k}}(1-r)\right)-\gamma_{n_{k}}\left(1-\delta_{n_{k}}\right)\left(1-\lambda_{n_{k}}\right)}\left\langle f\left(x^{*}\right)-x^{*}, J\left(y_{n_{k}}-x^{*}\right)\right\rangle \\
= & {\left[1-\frac{\left(1+\beta_{n_{k}}(1-r)\right)\left(1+\delta_{n_{k}}(1-r)\right)-\gamma_{n_{k}}\left(1-\delta_{n_{k}}\right)\left(1-\lambda_{n_{k}}\right)}{\left(1+\beta_{n_{k}}(1-r)\right)\left(1+\delta_{n_{k}}(1-r)\right)-\gamma_{n_{k}}\left(1-\delta_{n_{k}}\right)\left(1-\lambda_{n_{k}}\right)}\right.} \\
& \left.-\frac{-\left(1-\gamma_{n_{k}}-\beta_{n_{k}}(1-r)\right)\left(1+\delta_{n_{k}}(1-r)\right)-\gamma_{n_{k}}\left(r \delta_{n_{k}}+\left(1-\delta_{n_{k}}\right) \lambda_{n_{k}}\right)}{\left(1+\beta_{n_{k}}(1-r)\right)\left(1+\delta_{n_{k}}(1-r)\right)-\gamma_{n_{k}}\left(1-\delta_{n_{k}}\right)\left(1-\lambda_{n_{k}}\right)}\right]\left\|x_{n_{k}}-x^{*}\right\|^{2} \\
& +\frac{2 \beta_{n_{k}}\left(1+\delta_{n_{k}}(1-r)\right)}{\left(1+\beta_{n_{k}}(1-r)\right)\left(1+\delta_{n_{k}}(1-r)\right)-\gamma_{n_{k}}\left(1-\delta_{n_{k}}\right)\left(1-\lambda_{n_{k}}\right)}\left\langle f\left(x^{*}\right)-x^{*}, J\left(x_{n_{k+1}}-x^{*}\right)\right\rangle \\
& +\frac{2 \gamma_{n_{k}} \delta_{n_{k}}}{\left(1+\beta_{n_{k}}(1-r)\right)\left(1+\delta_{n_{k}}(1-r)\right)-\gamma_{n_{k}}\left(1-\delta_{n_{k}}\right)\left(1-\lambda_{n_{k}}\right)}\left\langle f\left(x^{*}\right)-x^{*}, J\left(y_{n_{k}}-x^{*}\right)\right\rangle,
\end{aligned}
$$

where

$$
\begin{aligned}
\alpha_{n_{k}}^{\prime}= & \frac{\left(1+\beta_{n_{k}}(1-r)\right)\left(1+\delta_{n_{k}}(1-r)\right)-\gamma_{n_{k}}\left(1-\delta_{n_{k}}\right)\left(1-\lambda_{n_{k}}\right)-\left(1-\gamma_{n_{k}}-\beta_{n_{k}}(1-r)\right)\left(1+\delta_{n_{k}}(1-r)\right)-\gamma_{n_{k}}\left(r \delta_{n_{k}}+\left(1-\delta_{n_{k}}\right) \lambda_{n_{k}}\right)}{\left(1+\beta_{n_{k}}(1-r)\right)\left(1+\delta_{n_{k}}(1-r)\right)-\gamma_{n_{k}}\left(1-\delta_{n_{k}}\right)\left(1-\lambda_{n_{k}}\right)} \\
= & \frac{2 \beta_{n_{k}}(1-r)\left(1+\delta_{n_{k}}(1-r)\right)+2 \gamma_{n_{k}} \delta_{n_{k}}(1-r)}{\left(1+\beta_{n_{k}}(1-r)\right)\left(1+\delta_{n_{k}}(1-r)\right)-\gamma_{n_{k}}\left(1-\delta_{n_{k}}\right)\left(1-\lambda_{n_{k}}\right)} \\
= & \frac{2\left(1-\alpha_{n_{k}}-\gamma_{n_{k}}\right)(1-r)\left(1+\delta_{n_{k}}(1-r)\right)+2 \gamma_{n_{k}} \delta_{n_{k}}(1-r)}{\left(1+\beta_{n_{k}}(1-r)\right)\left(1+\delta_{n_{k}}(1-r)\right)-\gamma_{n_{k}}\left(1-\delta_{n_{k}}\right)\left(1-\lambda_{n_{k}}\right)} \in(0,1)
\end{aligned}
$$

and

$$
\begin{aligned}
\delta_{n_{k}}^{\prime}= & \frac{2 \beta_{n_{k}}\left(1+\delta_{n_{k}}(1-r)\right)}{\left(1+\beta_{n_{k}}(1-r)\right)\left(1+\delta_{n_{k}}(1-r)\right)-\gamma_{n_{k}}\left(1-\delta_{n_{k}}\right)\left(1-\lambda_{n_{k}}\right)}\left\langle f\left(x^{*}\right)-x^{*}, J\left(x_{n_{k+1}}-x^{*}\right)\right\rangle \\
& +\frac{2 \gamma_{n_{k}} \delta_{n_{k}}}{\left(1+\beta_{n_{k}}(1-r)\right)\left(1+\delta_{n_{k}}(1-r)\right)-\gamma_{n_{k}}\left(1-\delta_{n_{k}}\right)\left(1-\lambda_{n_{k}}\right)}\left\langle f\left(x^{*}\right)-x^{*}, J\left(y_{n_{k}}-x^{*}\right)\right\rangle .
\end{aligned}
$$


Now, from the condition (iv), (3.5), (3.4), and Lemma 2.4, we have $\sum_{k=0}^{\infty} \alpha_{n_{k}}^{\prime}=\infty$ and $\underset{k \rightarrow \infty}{\limsup } \frac{\delta_{n_{k}}^{\prime}}{\alpha_{n_{k}}^{\prime}} \leqslant 0$. Then we get $\left\|x_{n_{k}}-x^{*}\right\| \rightarrow 0$ as $k \rightarrow \infty$. This is a contradiction, hence the sequence $\left\{x_{n}\right\}$ converges strongly to $x^{*} \in \mathrm{F}(\mathcal{S})$. The proof is complete.

Corollary 3.2. Let $\mathrm{C}$ be a nonempty closed convex subset of uniformly convex and uniformly smooth Banach space $\mathrm{E}$. Let $\mathrm{f}$ be a generalized contraction mapping from $\mathrm{C}$ into itself and $\mathrm{S}=\{\mathrm{T}(\mathrm{t}): \mathrm{t} \geqslant 0\}$ be a nonexpansive semigroup from $\mathrm{C}$ into itself such that $\mathrm{F}(\mathcal{S}) \neq 0$. Let $\left\{\mathrm{x}_{\mathrm{n}}\right\}$ be the sequences defined by $\mathrm{x}_{1} \in \mathrm{C}$ and

$$
\left\{\begin{array}{l}
z_{n}=\frac{x_{n}+x_{n+1}}{2} \\
y_{n}=\delta_{n} f\left(x_{n}\right)+\left(1-\delta_{n}\right) z_{n} \\
x_{n+1}=\alpha_{n} x_{n}+\beta_{n} f\left(x_{n}\right)+\gamma_{n} T\left(\mu_{n}\right) y_{n}
\end{array}\right.
$$

where $\left\{\alpha_{n}\right\},\left\{\beta_{n}\right\},\left\{\gamma_{n}\right\}$, and $\left\{\delta_{n}\right\}$ are the sequences in $(0,1)$. The following conditions are satisfied:

(i) $\alpha_{n}+\beta_{n}+\gamma_{n}=1$;

(ii) $\lim _{n \rightarrow \infty} \beta_{n}=\lim _{n \rightarrow \infty} \delta_{n}=\lim _{n \rightarrow \infty} \mu_{n}=\lim _{n \rightarrow \infty}\left|\alpha_{n+1}-\alpha_{n}\right|=0$ and $\lim _{n \rightarrow \infty} \sup _{x \in \tilde{C}}\left\|\mathrm{~T}\left(\mu_{n+1}\right) x-\mathrm{T}\left(\mu_{n}\right) x\right\|=0$, where $\tilde{\mathrm{C}}$ is bounded subset of $\mathrm{C}$;

(iii) $0<\liminf _{n \rightarrow \infty} \alpha_{n} \leqslant \limsup _{n \rightarrow \infty} \alpha_{n}<1$;

(iv) $\sum_{n=0}^{\infty} \beta_{n}=\infty$ for all $n \geqslant 1$.

Then $\left\{x_{n}\right\}$ converges strongly to $x^{*} \in F(\mathcal{S})$ which also solves the following variational inequality:

$$
\left\langle(\mathrm{I}-\mathrm{f}) \mathrm{x}^{*}, \mathrm{~J}\left(z-x^{*}\right)\right\rangle \leqslant 0, \forall z \in \mathrm{F}(\mathcal{S}) .
$$

Proof. Putting $\lambda_{n}=\frac{1}{2}$ in Theorem 3.1, we can conclude the desired conclusion easily. This completes the proof.

\section{Applications}

4.1. Application to the other forms of semigroups

Theorem 4.1. Let $\mathrm{C}$ be a nonempty closed convex subset of uniformly convex and uniformly smooth Banach space $\mathrm{E}$. Let $\mathrm{f}$ be a generalized contraction mapping from $\mathrm{C}$ into itself and $\mathrm{S}=\{\mathrm{T}(\mathrm{t}): \mathrm{t} \geqslant 0\}$ be a nonexpansive semigroup from $\mathrm{C}$ into itself such that $\mathrm{F}(\mathcal{S}) \neq 0$. Let $\left\{\mathrm{x}_{\mathrm{n}}\right\}$ be the sequences defined by $\mathrm{x}_{1} \in \mathrm{C}$ and

$$
\left\{\begin{array}{l}
z_{n}=\lambda_{n} x_{n}+\left(1-\lambda_{n}\right) x_{n+1} \\
y_{n}=\delta_{n} f\left(x_{n}\right)+\left(1-\delta_{n}\right) z_{n}, \\
x_{n+1}=\alpha_{n} x_{n}+\beta_{n} f\left(x_{n}\right)+\gamma_{n} \frac{1}{t_{n}} \int_{0}^{t_{n}} T(t) y_{n} d t
\end{array}\right.
$$

where $\left\{\alpha_{n}\right\},\left\{\beta_{n}\right\},\left\{\gamma_{n}\right\},\left\{\delta_{n}\right\}$, and $\left\{\lambda_{n}\right\}$ are the sequences in $(0,1),\left\{t_{n}\right\}$ is an increasing sequence in $(0, \infty)$ such that $\lim _{n \rightarrow \infty} t_{n}=\infty$, and $\lim _{n \rightarrow \infty} \frac{t_{n}}{t_{n+1}}=1$. The following conditions are satisfied:

(i) $\alpha_{n}+\beta_{n}+\gamma_{n}=1$;

(ii) $\lim _{n \rightarrow \infty} \beta_{n}=\lim _{n \rightarrow \infty} \delta_{n}=\lim _{n \rightarrow \infty} \mu_{n}=\lim _{n \rightarrow \infty}\left|\alpha_{n+1}-\alpha_{n}\right|=\lim _{n \rightarrow \infty}\left|\lambda_{n+1}-\lambda_{n}\right|=0$ and

$$
\lim _{n \rightarrow \infty} \sup _{x \in \tilde{C}}\left\|\frac{1}{t_{n+1}} \int_{0}^{t_{n+1}} T(t) x d s-\frac{1}{t_{n}} \int_{0}^{t_{n}} T(t) x d s\right\|=0,
$$

where $\tilde{\mathrm{C}}$ is bounded subset of $\mathrm{C}$; 
(iii) $0<\liminf _{n \rightarrow \infty} \alpha_{n} \leqslant \limsup _{n \rightarrow \infty} \alpha_{n}<1$;

(iv) $\sum_{n=0}^{\infty} \beta_{n}=\infty$ for all $n \geqslant 1$.

Then $\left\{x_{n}\right\}$ converges strongly to $x^{*} \in \mathrm{F}(\mathcal{S})$ which also solves the following variational inequality:

$$
\left\langle(\mathrm{I}-\mathrm{f}) x^{*}, \mathrm{~J}\left(z-x^{*}\right)\right\rangle \leqslant 0, \forall z \in \mathrm{F}(\mathcal{S}) .
$$

Proof. Define $\mu_{n}(f)=\frac{1}{t_{n}} \int_{0}^{t_{n}} f(t) d t, \forall n=0,1,2, \ldots$, where $f$ belong to the space of all real valued bounded continuous functions on positive real number with supremum norm. Then, $\left\{\mu_{n}\right\}$ is a regular sequence of means [1]. Further, we have $T\left(\mu_{n}\right) x=\frac{1}{t_{n}} \int_{0}^{t_{n}} T(t) x d t, \forall x \in C$ and apply Theorem 3.1 to conclude the result.

Corollary 4.2. Let $\mathrm{C}$ be a nonempty closed convex subset of uniformly convex and uniformly smooth Banach space $\mathrm{E}$. Let $\mathrm{f}$ be a generalized contraction mapping from $\mathrm{C}$ into itself and $\mathcal{S}=\{\mathrm{T}(\mathrm{t}): \mathrm{t} \geqslant 0\}$ be a nonexpansive semigroup from $\mathrm{C}$ into itself such that $\mathrm{F}(\mathcal{S}) \neq 0$. Let $\left\{x_{n}\right\}$ be the sequences defined by $x_{1} \in \mathrm{C}$ and

$$
\left\{\begin{array}{l}
z_{n}=\frac{x_{n}+x_{n+1}}{2} \\
y_{n}=\delta_{n} f\left(x_{n}\right)+\left(1-\delta_{n}\right) z_{n} \\
x_{n+1}=\alpha_{n} x_{n}+\beta_{n} f\left(x_{n}\right)+\gamma_{n} \frac{1}{t_{n}} \int_{0}^{t_{n}} T(t) y_{n} d t
\end{array}\right.
$$

where $\left\{\alpha_{n}\right\},\left\{\beta_{n}\right\},\left\{\gamma_{n}\right\},\left\{\delta_{n}\right\}$, and $\left\{\lambda_{n}\right\}$ are the sequences in $(0,1),\left\{t_{n}\right\}$ is an increasing sequence in $(0, \infty)$ such that $\lim _{n \rightarrow \infty} t_{n}=\infty$ and $\lim _{n \rightarrow \infty} \frac{t_{n}}{t_{n+1}}=1$. The following conditions are satisfied:

(i) $\alpha_{n}+\beta_{n}+\gamma_{n}=1$;

(ii) $\lim _{n \rightarrow \infty} \beta_{n}=\lim _{n \rightarrow \infty} \delta_{n}=\lim _{n \rightarrow \infty} \mu_{n}=\lim _{n \rightarrow \infty}\left|\alpha_{n+1}-\alpha_{n}\right|=\lim _{n \rightarrow \infty}\left|\lambda_{n+1}-\lambda_{n}\right|=0$ and

$$
\lim _{n \rightarrow \infty} \sup _{x \in \tilde{C}}\left\|\frac{1}{t_{n+1}} \int_{0}^{t_{n+1}} T(t) x d s-\frac{1}{t_{n}} \int_{0}^{t_{n}} T(t) x d s\right\|=0,
$$

where $\tilde{\mathrm{C}}$ is bounded subset of $\mathrm{C}$;

(iii) $0<\liminf _{n \rightarrow \infty} \alpha_{n} \leqslant \limsup _{n \rightarrow \infty} \alpha_{n}<1$;

(iv) $\sum_{n=0}^{\infty} \beta_{n}=\infty$ for all $n \geqslant 1$.

Then $\left\{x_{n}\right\}$ converges strongly to $x^{*} \in F(\mathcal{S})$ which also solves the following variational inequality:

$$
\left\langle(\mathrm{I}-\mathrm{f}) \mathrm{x}^{*}, \mathrm{~J}\left(z-x^{*}\right)\right\rangle \leqslant 0, \forall z \in \mathrm{F}(\mathcal{S}) .
$$

\subsection{Application to Hilbert spaces.}

From the duality mapping $\mathrm{J}: \mathrm{E} \rightarrow 2^{\mathrm{E}^{*}}$, if $\mathrm{E}=\mathrm{H}$ is a real Hilbert space then $\mathrm{J}=\mathrm{I}$ is the identity mapping.

Theorem 4.3. Let $\mathrm{C}$ be a nonempty closed convex subset of a Hilbert space $\mathrm{H}$. Let $\mathrm{f}$ be a generalized contraction mapping from $\mathrm{C}$ into itself and $\mathcal{S}=\{\mathrm{T}(\mathrm{t}): \mathrm{t} \geqslant 0\}$ be a nonexpansive semigroup from $\mathrm{C}$ into itself such that $\mathrm{F}(\mathcal{S}) \neq 0$. Let $\left\{x_{n}\right\}$ be the sequences defined by $x_{1} \in \mathrm{C}$ and

$$
\left\{\begin{array}{l}
z_{n}=\lambda_{n} x_{n}+\left(1-\lambda_{n}\right) x_{n+1} \\
y_{n}=\delta_{n} f\left(x_{n}\right)+\left(1-\delta_{n}\right) z_{n} \\
x_{n+1}=\alpha_{n} x_{n}+\beta_{n} f\left(x_{n}\right)+\gamma_{n} T\left(\mu_{n}\right) y_{n},
\end{array}\right.
$$

where $\left\{\alpha_{n}\right\},\left\{\beta_{n}\right\},\left\{\gamma_{n}\right\},\left\{\delta_{n}\right\}$, and $\left\{\lambda_{n}\right\}$ are the sequences in $(0,1)$. The following conditions are satisfied: 
(i) $\alpha_{n}+\beta_{n}+\gamma_{n}=1$;

(ii) $\lim _{n \rightarrow \infty} \beta_{n}=\lim _{n \rightarrow \infty} \delta_{n}=\lim _{n \rightarrow \infty} \mu_{n}=\lim _{n \rightarrow \infty}\left|\alpha_{n+1}-\alpha_{n}\right|=\lim _{n \rightarrow \infty}\left|\lambda_{n+1}-\lambda_{n}\right|=0$ and $\lim _{n \rightarrow \infty} \sup _{x \in \tilde{C}} \| T\left(\mu_{n+1}\right) x-$ $\mathrm{T}\left(\mu_{\mathrm{n}}\right) \mathrm{x} \|=0$, where $\tilde{\mathrm{C}}$ is bounded subset of $\mathrm{C}$;

(iii) $0<\liminf _{n \rightarrow \infty} \alpha_{n} \leqslant \limsup _{n \rightarrow \infty} \alpha_{n}<1$;

(iv) $\sum_{n=0}^{\infty} \beta_{n}=\infty$ for all $n \geqslant 1$.

Then $\left\{x_{n}\right\}$ converges strongly to $x^{*} \in F(\mathcal{S})$ which also solves the following variational inequality:

$$
\left\langle(I-f) x^{*}, z-x^{*}\right\rangle \leqslant 0, \forall z \in F(\mathcal{S}) .
$$

Corollary 4.4. Let $\mathrm{C}$ be a nonempty closed convex subset of a Hilbert space $\mathrm{H}$. Let $\mathrm{f}$ be a generalized contraction mapping from $\mathrm{C}$ into itself and $\mathcal{S}=\{\mathrm{T}(\mathrm{t}): \mathrm{t} \geqslant 0\}$ be a nonexpansive semigroup from $\mathrm{C}$ into itself such that $\mathrm{F}(\mathcal{S}) \neq 0$. Let $\left\{x_{n}\right\}$ be the sequences defined by $x_{1} \in \mathrm{C}$ and

$$
\left\{\begin{array}{l}
z_{n}=\frac{x_{n}+x_{n+1}}{2} \\
y_{n}=\delta_{n} f\left(x_{n}\right)+\left(1-\delta_{n}\right) z_{n} \\
x_{n+1}=\alpha_{n} x_{n}+\beta_{n} f\left(x_{n}\right)+\gamma_{n} T\left(\mu_{n}\right) y_{n}
\end{array}\right.
$$

where $\left\{\alpha_{n}\right\},\left\{\beta_{n}\right\},\left\{\gamma_{n}\right\}$, and $\left\{\delta_{n}\right\}$ are the sequences in $(0,1)$. The following conditions are satisfied:

(i) $\alpha_{n}+\beta_{n}+\gamma_{n}=1$;

(ii) $\lim _{n \rightarrow \infty} \beta_{n}=\lim _{n \rightarrow \infty} \delta_{n}=\lim _{n \rightarrow \infty} \mu_{n}=\lim _{n \rightarrow \infty}\left|\alpha_{n+1}-\alpha_{n}\right|=0$ and $\lim _{n \rightarrow \infty} \sup _{x \in \tilde{C}}\left\|T\left(\mu_{n+1}\right) x-T\left(\mu_{n}\right) x\right\|=0$, where C bounded subset of $\mathrm{C}$;

(iii) $0<\liminf _{n \rightarrow \infty} \alpha_{n} \leqslant \limsup _{n \rightarrow \infty} \alpha_{n}<1$;

(iv) $\sum_{n=0}^{\infty} \beta_{n}=\infty$ for all $n \geqslant 1$.

Then $\left\{x_{n}\right\}$ converges strongly to $x^{*} \in F(\mathcal{S})$ which also solves the following variational inequality:

$$
\left\langle(I-f) x^{*}, z-x^{*}\right\rangle \leqslant 0, \forall z \in F(\mathcal{S}) .
$$

\section{Numerical example}

Example 5.1. Let $E=\mathbb{R}^{3}$ and an inner product $\langle\cdot, \cdot\rangle: \mathbb{R}^{3} \times \mathbb{R}^{3} \rightarrow \mathbb{R}$ be defined by

$$
\langle\mathbf{x}, \mathbf{y}\rangle=\mathbf{x} \cdot \mathbf{y}=x^{1} \cdot y^{1}+x^{2} \cdot y^{2}+x^{3} \cdot y^{3}, \quad \forall \mathbf{x}=\left(x^{1}, x^{2}, x^{3}\right), \mathbf{y}=\left(y^{1}, y^{2}, y^{3}\right)
$$

and the usual norm $\|\cdot\|: \mathbb{R}^{3} \rightarrow \mathbb{R}$ be defined by

$$
\|\mathbf{x}\|=\sqrt{\left(x^{1}\right)^{2}+\left(x^{2}\right)^{2}+\left(x^{3}\right)^{2}} .
$$

Let $C=\left\{\mathbf{x} \in \mathbb{R}^{3} \mid\|\mathbf{x}\| \leqslant 1\right\}, \alpha_{n}=\lambda_{n}=\frac{n+1}{3 n}, \beta_{n}=\frac{1}{3 n}, \delta_{n}=\mu_{n}=\frac{1}{n}, \gamma_{n}=\frac{2 n-2}{3 n}$, and $x_{1}=(1,2,3)$ for all $n=1,2, \ldots$, which satisfy the conditions (I)-(IV) in Theorem 3.1. We define the mappings $T(t), f$ : $\mathbb{R}^{3} \rightarrow \mathbb{R}^{3}$ as follows: $(\mathrm{T}(\mathrm{t}))(\mathbf{x})=\mathbf{x} e^{-\mathrm{t}}$ and $\mathrm{f}(\mathbf{x})=\frac{\mathbf{x}}{10}$ for all $\mathbf{x} \in \mathbb{R}^{3}$. Then the sequence

$$
\mathbf{x}_{n+1}=\frac{30 n^{3}+33 n^{2}+\left(20 n^{3}-14 n^{2}-26 n+20\right) e^{-1 / n}}{90 n^{3}-\left(40 n^{3}-100 n^{2}+80 n-20\right) e^{-1 / n}} x_{n}
$$

converges strongly to $\mathbf{0}=(0,0,0)$, which is shown in Figure 1 and Table 1 . 

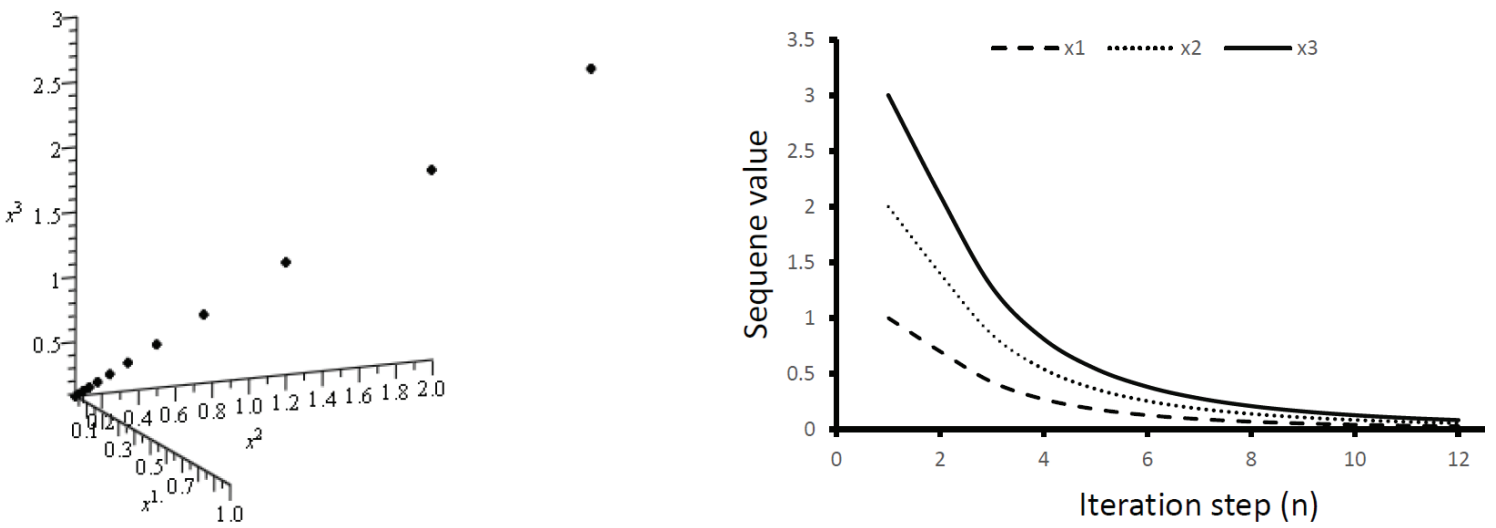

Figure 1: The iteration process.

Table 1: The value of sequence $\left\{\mathbf{x}_{n}\right\}$.

\begin{tabular}{|c|c|c|c|c|c|c|c|}
\hline $\mathrm{n}$ & $x_{\mathrm{n}}^{1}$ & $x_{\mathrm{n}}^{2}$ & $\chi_{n}^{3}$ & $\mathrm{n}$ & $\chi_{\mathfrak{n}}^{1}$ & $\chi_{\mathfrak{n}}^{2}$ & $\chi_{n}^{3}$ \\
\hline 1 & 1 & 2 & 3 & 10 & 0.042547 & 0.085093 & 0.127640 \\
2 & 0.7 & 1.4 & 2.1 & 50 & 0.000722 & 0.001445 & 0.002167 \\
3 & 0.425637 & 0.851275 & 1.276912 & 100 & 0.000109 & 0.000218 & 0.000327 \\
4 & 0.270485 & 0.540970 & 0.811455 & 400 & 0.0000023 & 0.0000046 & 0.0000069 \\
5 & 0.181409 & 0.362819 & 0.544228 & 797 & 0.0000003 & 0.0000006 & 0.00000099 \\
\hline
\end{tabular}

\section{Acknowledgment}

The authors would like to thank the editors and the referees for their insightful comments. This research was supported by the Rajamangala University of Technology Lanna Tak (RMUTL Tak).

\section{References}

[1] S. Atsushiba, W. Takahashi, Strong convergence of Mann's-type iterations for nonexpansive semigroups in general Banach spaces, Nonlinear Anal., 61 (2005), 881-899. 4.1

[2] J.-P. Gossez, E. Lami Dozo, Some geometric properties related to the fixed point theory for nonexpansive mappings, Pacific J. Math., 40 (1972), 565-573. 2

[3] W.-B. Guan, An iterative method for variational inequality problems, J. Inequal. Appl., 2013 (2013), 10 pages. 1

[4] Y. Ke, C. Ma, The generalized viscosity implicit rules of nonexpansive mappings in Hilbert spaces, Fixed Point Theory Appl., 2015 (2015), 21 pages. 1

[5] A. T.-M. Lau, Amenability and fixed point property for semigroup of nonexpansive mapping, Fixed point theory Appl. (Marseille, (1989)), Pitman Res. Notes Math. Ser. Longman Sci. Tech., Harlow, (1991). 1

[6] A. T.-M. Lau, Y. Zhang, Fixed point properties of semigroups of non-expansive mappings, J. Funct. Anal., 254 (2008), 2534-2554. 1

[7] L.-C. Lim, On characterizations of Meir-Keeler contractive maps, Nonlinear Anal., 46 (2001), 113-120. 1.3

[8] P. Sunthrayuth, P. Kumam, Viscosity approximation methods based on generalized contraction mappings for a countable family of strict pseudo-contractions, a general system of variational inequalities and a generalized mixed equilibrium problem in Banach spaces, Math. Comput. Modelling, 58 (2013), 1814-1828. 2.2, 2.3

[9] T. Suzuki, Strong convergence of Krasnoselskii and Mann's type sequences for one-parameter nonexpansive semigroups without Bochner integrals, J. Math. Anal. Appl., 305 (2005), 227-239. 2.1

[10] T. Suzuki, Moudafi's viscosity approximations with MeirKeeler contractions, J. Math. Anal. Appl., 325 (2007), $342-352$. 1.4

[11] Y. Wang, H.-K. Xu, Hybrid method for a class of accretive variational inequalities involving nonexpansive mappings, J. Inequal. Appl., 2014 (2014), 9 pages. 1

[12] H.-K. Xu, Iterative algorithms for nonlinear operators, J. London Math. Soc., 66 (2002), 240-256. 2.4

[13] H.-K. Xu, M. A. Alghamdi, N. Shahzad, The viscosity technique for the implicit midpoint rule of nonexpansive mappings in Hilbert spaces, Fixed Point Theory Appl., 2015 (2015), 12 pages. 1

[14] Q. Yan, G. Cai, P. Luo, Strong convergence theorems for the generalized viscosity implicit rules of nonexpansive mappings in uniformly smooth Banach spaces, J. Nonlinear Sci. Appl., 9 (2016), 4039-4051. 1 